\title{
Structure of turbulent flow over regular arrays of cubical roughness
}

\author{
O. COCEAL ${ }^{1}$, A. DOBRE ${ }^{1}$, T. G. THOMAS \\ AND S. E. BELCHER ${ }^{1}$ \\ ${ }^{1}$ Department of Meteorology, University of Reading, Reading, RG6 6BB, UK \\ ${ }^{2}$ School of Engineering Sciences, University of Southampton, Southampton SO17 1BJ, UK
}

(Received 11 May 2006 and in revised form 13 June 2007)

The structure of turbulent flow over large roughness consisting of regular arrays of cubical obstacles is investigated numerically under constant pressure gradient conditions. Results are analysed in terms of first- and second-order statistics, by visualization of instantaneous flow fields and by conditional averaging. The accuracy of the simulations is established by detailed comparisons of first- and second-order statistics with wind-tunnel measurements. Coherent structures in the log region are investigated. Structure angles are computed from two-point correlations, and quadrant analysis is performed to determine the relative importance of Q2 and Q4 events (ejections and sweeps) as a function of height above the roughness. Flow visualization shows the existence of low-momentum regions (LMRs) as well as vortical structures throughout the log layer. Filtering techniques are used to reveal instantaneous examples of the association of the vortices with the LMRs, and linear stochastic estimation and conditional averaging are employed to deduce their statistical properties. The conditional averaging results reveal the presence of LMRs and regions of Q2 and Q4 events that appear to be associated with hairpin-like vortices, but a quantitative correspondence between the sizes of the vortices and those of the LMRs is difficult to establish; a simple estimate of the ratio of the vortex width to the LMR width gives a value that is several times larger than the corresponding ratio over smooth walls. The shape and inclination of the vortices and their spatial organization are compared to recent findings over smooth walls. Characteristic length scales are shown to scale linearly with height in the log region. Whilst there are striking qualitative similarities with smooth walls, there are also important differences in detail regarding: (i) structure angles and sizes and their dependence on distance from the rough surface; (ii) the flow structure close to the roughness; (iii) the roles of inflows into and outflows from cavities within the roughness; (iv) larger vortices on the rough wall compared to the smooth wall; (v) the effect of the different generation mechanism at the wall in setting the scales of structures.

\section{Introduction}

It is now widely accepted that large-scale organized structures play a crucial role in the dynamics of turbulent shear flows (e.g. Robinson 1991; Panton 2001). Detailed knowledge of the coherent structure topology and dynamics can lead to two important benefits: the development of low-dimensional flow dynamics models (Aubry et al. 1988) and solutions for efficient flow control strategies (Gad el Hak 
2000). Hence there is much interest in detecting and analysing coherent structures from both a theoretical and a practical point of view.

The structure and dynamics of turbulent flow over smooth walls has been extensively studied (see the reviews by Cantwell 1981, Robinson 1991 and Panton 2001). However, relatively little is known about flows over rough walls, as emphasized in the recent review by Jimenez (2004), despite the enormous significance of these flows for problems in engineering and atmospheric applications. There are two overlapping layers over smooth walls: an inner layer where viscous processes dominate, and an outer layer far from these effects. Following Pope (2000), the inner layer is the region where $z / \delta<0.1$ and the outer layer is where $z^{+}>50$. Here, $z$ is wall-normal distance, $\delta$ is boundary layer height and the + superscript denotes distance in wall units (normalized by the viscous length scale $v / u_{\tau}$, where $v$ is kinematic viscosity and $u_{\tau}$ is the wall friction velocity). For sufficiently high Reynolds number, an inertial sublayer or log layer exists, roughly in the region $z^{+}>30, z / \delta<0.3$. The viscous sublayer is the region $z^{+}<5$, and the buffer layer is the region between the viscous sublayer and the log layer, $5<z^{+}<30$. Over rough walls a $\log$ region also exists in the outer layer, but the near-wall flow is spatially inhomogeneous and depends on the geometry of the roughness. The roughness sublayer is then the region immediately below the log layer. Over very rough walls, the viscous sublayer is effectively replaced by the roughness sublayer (Raupach, Antonia \& Rajagopalan 1991); it is the roughness itself, rather than any viscous layers attached to it, that provides the dominant mechanism for generating near-wall turbulence.

In their well-known review on rough-wall boundary layers, Raupach et al. (1991) argued that wall-bounded flows have universal characteristics in the outer layer, and that the structure of the outer boundary layer over a rough wall is similar to that over a smooth wall, even though the production mechanisms near the wall are very different. There is now some disagreement in the literature as to what extent this is true. It has been suggested that some particular types of roughness ('d type') may influence the flow across the whole boundary layer. These typically consist of two-dimensional bar roughness, and have been the subject of detailed laboratory and numerical investigations over a number of years (Djenidi, Elavarasan \& Antonia 1999; Leonardi et al. 2003, 2004). Surprisingly little detailed information exists on roughness consisting of large three-dimensional obstacles, despite its obvious practical relevance to many engineering and atmospheric flows. Clearly, detailed investigations over more generic, three-dimensional roughness are needed.

\subsection{Coherent structures over smooth walls}

Since the pioneering work of Kline et al. (1967), there has been a large amount of work on turbulent structures over smooth walls, building upon important early experimental and numerical developments (Kim, Kline \& Reynolds 1971; Kim, Moin \& Moser 1987). Much attention has focused on the inner and buffer layers, especially with regard to the role of near-wall low-speed streaks. Less effort has been devoted to investigations of structure in the outer layer of wall-bounded flows. Recently Adrian, Meinhart \& Tomkins (2000) and Tomkins \& Adrian (2003) convincingly demonstrated using particle image velocimetry (PIV) measurements that streaky structures, or lowmomentum regions (LMRs), exist throughout the log region over a smooth wall. The length and width of the LMRs was shown to increase linearly with distance from the wall in the log layer, which they interpreted as supporting Townsend's attached-eddy hypothesis (Townsend 1976). By means of flow visualization and low-pass filtering the authors demonstrated that the LMRs are bordered by hairpin vortices organized 
streamwise into coherent packets. These observations led the authors to suggest a 'parent-offspring' vortex regeneration mechanism whereby an existing hairpin vortex spawns new hairpins both upstream and downstream, based on direct numerical simulations (DNS) of the evolution of a hairpin vortex by Zhou et al. (1999). Thence, an elongated LMR is induced bordered by the hairpin legs. Tomkins \& Adrian (2003) further proposed a vortex merging mechanism that leads to self-similar growth of interacting hairpin vortices, and thus explains the linear scaling of characteristic sizes of the vortices and LMRs in the log layer. The reader is referred to the excellent introduction in the paper by Adrian et al. (2000) for a review of hairpin vortices in the boundary layer, and to the concluding section of the paper, in which they summarize the evidence for their hairpin vortex packet model.

\subsection{Coherent structures over rough walls}

A key question is how the presence of roughness elements on the wall modifies the structures. Early investigations by Grass (1971) over smooth and rough surfaces in a water channel using sand and pebbles as roughness elements showed the existence of near-wall streaky structures over these rough surfaces. Grass pointed out the strong effects of inrushes and ejections of fluid from cavities within the roughness on the structures. More recent flow visualizations by Grass, Stuart \& Mansour-Thehrani (1993) over spheres showed that the distance between the streaks was increased and their streamwise coherence reduced relative to a smooth wall.

Krogstad \& Antonia (1994) made measurements in a wind tunnel over mesh roughness (k-type) and Djenidi et al. (1999) over square bar roughness (d-type). They found that for the mesh roughness there was a slight increase in the spanwise size of the structures, whereas the normalized spacing in the case of the d-type roughness was the same as for a smooth wall. Djenidi at al. (1999) found that strong outflows and inflows occurred over transverse square cavities in a pseudo-random manner, and attributed them to the passage of near-wall quasi-streamwise vortices. They also used flow visualization to estimate the size of the associated low-speed streaks and found that the mean spanwise spacing between the streaks in wall units, $\lambda^{+}$, was about 100 , similar to the smooth wall value. Recently Leonardi et al. (2004) performed DNS investigating the near-wall structure of turbulent channel flow over square bars for different packing densities. They concluded that relative to smooth walls the structures appear shorter in the streamwise direction but larger in the spanwise direction, and suggested that the changes may be related to the strengths of ejections of fluid from the cavities.

Very recently, Castro, Cheng \& Reynolds (2006) analysed single- and two-point statistics for turbulent flow over a staggered array of cubes. They showed that the dominant scales in the roughness sublayer are of the same order as the height of the cubes, but that there is a two-scale behaviour near the roughness top which they interpreted as the intermingling of shear-layer structures with much larger-scale structures. They also found that the average structure angle falls with height through the roughness sublayer, in contrast to the behaviour of smooth-wall boundary layers. These results emphasized the need for a deeper understanding of rough-wall flows.

A detailed and systematic investigation of the turbulence structure in the log region over rough walls, using eduction techniques like those employed by Adrian et al. (2000) and Tomkins \& Adrian (2003) remains to be done.

\subsection{The present work: questions and objectives}

In this paper we perform direct numerical simulations to elucidate the phenomenology of dominant coherent structures in the log layer above three-dimensional roughness 


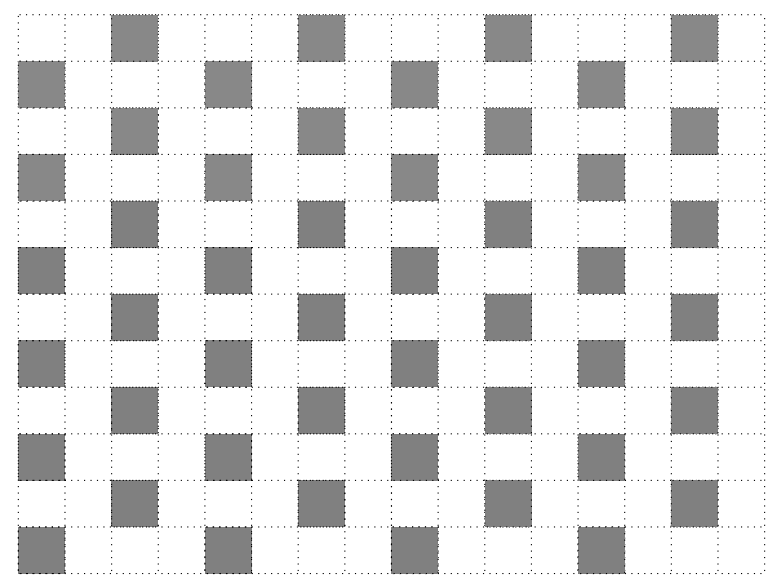

FIgURE 1. Plan view of computational domain. Height of domain is $H=8 h$, where $h$ is the cube height. Mean flow is from left to right. Periodic boundary conditions are imposed in the streamwise and lateral directions.

comprised of a regular array of cubes. A systematic study is undertaken by computing a number of statistics, performing flow visualization of instantaneous snapshots and analysing the data using conditional sampling and averaging. The results are examined in the light of similar findings over smooth walls and previous work over other types of roughness.

The rest of the paper is organized as follows. Section 2 outlines the numerical method. In $\S 3$, first- and second-order statistics are computed and comparisons are made with wind tunnel measurements in the literature. Two-point correlations and quadrant analysis are used to provide structural information. Section 4 describes coherent structures - low-momentum regions and vortices - observed using flow visualization and filtering techniques. Conditional averaging and linear stochastic estimation are then applied to compute statistical properties of the coherent structures. Section 5 concludes with a discussion of the main findings.

\section{Numerical procedure}

The Navier-Stokes equations are discretized using second-order central finite differences in space and a second-order Adams-Bashforth scheme in time, based on the pressure correction method. The code is parallelized in a highly efficient manner using MPI, and a flexible multi-block mapping strategy is developed to deal with the flow domain containing the complex geometry. The Poisson equation for pressure $p$ is solved by a multigrid method. For further details of numerical methods see Yao et al. (2001).

Figure 1 shows a plan view of the computational domain, which has streamwise, lateral and vertical dimensions $L=16 h, W=12 h$ and $H=8 h$ respectively $(16 h \times 12 h \times 8 h)$, where $h$ is the cube height. The array is staggered in the streamwise direction. Periodic boundary conditions are imposed in both streamwise and lateral directions to simulate an infinite array. The notational convention adopted is that $x, y$ and $z$ denote the streamwise, lateral and vertical coordinates, and that $u, v$ and $w$ denote the streamwise, lateral and vertical components of velocity respectively. The origin of coordinates is taken as the bottom left-hand corner of the domain. The present choice of domain size was guided by the results of wind tunnel measurements 
made by Castro et al. (2006) over the same geometry. They computed integral length scales $L_{x}, L_{y}$ and $L_{z}$ above the array of cubes from two-point correlations and found that $L_{x}$ is around $3 h$ and $L_{y}$ and $L_{z}$ are around $h$ in the inertial sublayer. The domain dimensions used here are thus much larger than these integral length scales.

The domain height $H=8 \mathrm{~h}$ is comparable with the boundary layer height $\delta=7.5 \mathrm{~h}$ in the wind-tunnel experiment of Cheng \& Castro (2002) and Castro et al. (2006), with whose results statistics computed from the present simulations are compared in $\S 3$. Whilst this ratio $\delta / h$ may appear small from the point of view of engineering applications (Jimenez 2004), it is not uncommon in a meteorological context such as over cities or forests (Rotach 1993; Cheng \& Castro 2002; Finnigan 2000). From a general fluid mechanics perspective, $\delta / h$ is a parameter and, given present computational resources, it is only currently feasible to perform a DNS for a relatively modest value of this parameter. Nevertheless, the present simulation is valuable as a study of turbulence structure in the somewhat extreme case of a 'very rough' surface composed of large roughness elements. The explicit comparison with the opposite extreme of a dynamically smooth wall given in the paper sets an interesting context within which to analyse flows that may come between these two extremes.

A free-slip boundary condition is applied at the top of the domain. No-slip is imposed on the bottom wall and on all cube surfaces. The flow is maintained by a height-independent streamwise pressure gradient of magnitude $u_{\tau}^{2} / H$, where $u_{\tau}$ is the total wall friction velocity. The Reynolds number of the flow, based on the velocity at the top of the domain and the cube height, is $R e=5800$. The roughness Reynolds number is $R e_{\tau} \equiv u_{\tau} h / v=500$. To ensure temporal convergence, the simulations were run for an initial duration of about $100 T$, where $T=h / u_{\tau}$ is an eddy turnover time for the largest eddies shed by the cubes. Statistics were collected and averaged over a further duration of $100 T$. The runs took 384 hours using 124 processors on an SGI Altix 3700 supercomputer, an equivalent of about 48000 single processor cpu hours.

An isotropic and uniform Cartesian grid was used with grid size $\Delta=h / 32$, with the first gridpoint from the cube surfaces being at a distance of $\Delta / 2$. In $\S 3$ the Kolmogorov length scale $\eta$ is computed from the energy spectrum at several locations above the array. The results show that $\eta$ increases monotonically from $0.0056 h$ at $z=h$ to $0.0116 h$ at $z=4.75 h$ (see figure $8 b$ ). This corresponds to the grid resolution lying in the range $2.7 \eta<\Delta<5.5 \eta$. Estimates give similar values of $\Delta \approx 4.2 \eta$ within the array. As pointed out by Moin \& Mahesh (1998), the smallest length scale that must be resolved depends on the energy spectrum and is typically greater than the Kolmogorov length scale $\eta$. For example in curved channel flow most of the dissipation occurs at scales greater than $15 \eta$ (Moin \& Mahesh 1998), whereas a calculation based on the Pao spectrum (Pao 1965) gives a dissipation cutoff of $11 \eta$. Based on these figures, the grid resolution is fine enough to capture most of the dissipation.

The grid resolution used here is coarser than in DNS studies over smooth walls (e.g. Kim et al. 1987), especially close to the walls, although it is comparable with the resolution in the rough-wall DNS of Leonardi et al. (2003). With the available computational resources and numerical methods it is not possible to match the resolution of the smooth-wall DNS near the solid walls. Hence, the thin boundary layers on individual cube facets may not be completely resolved, and one needs to consider what effect this may have on the flow. Grid resolution tests were performed by running a simulation at double the resolution $(\delta=h / 64)$. Because of the prohibitive computational cost, it was not possible to do this on the same size of domain. 

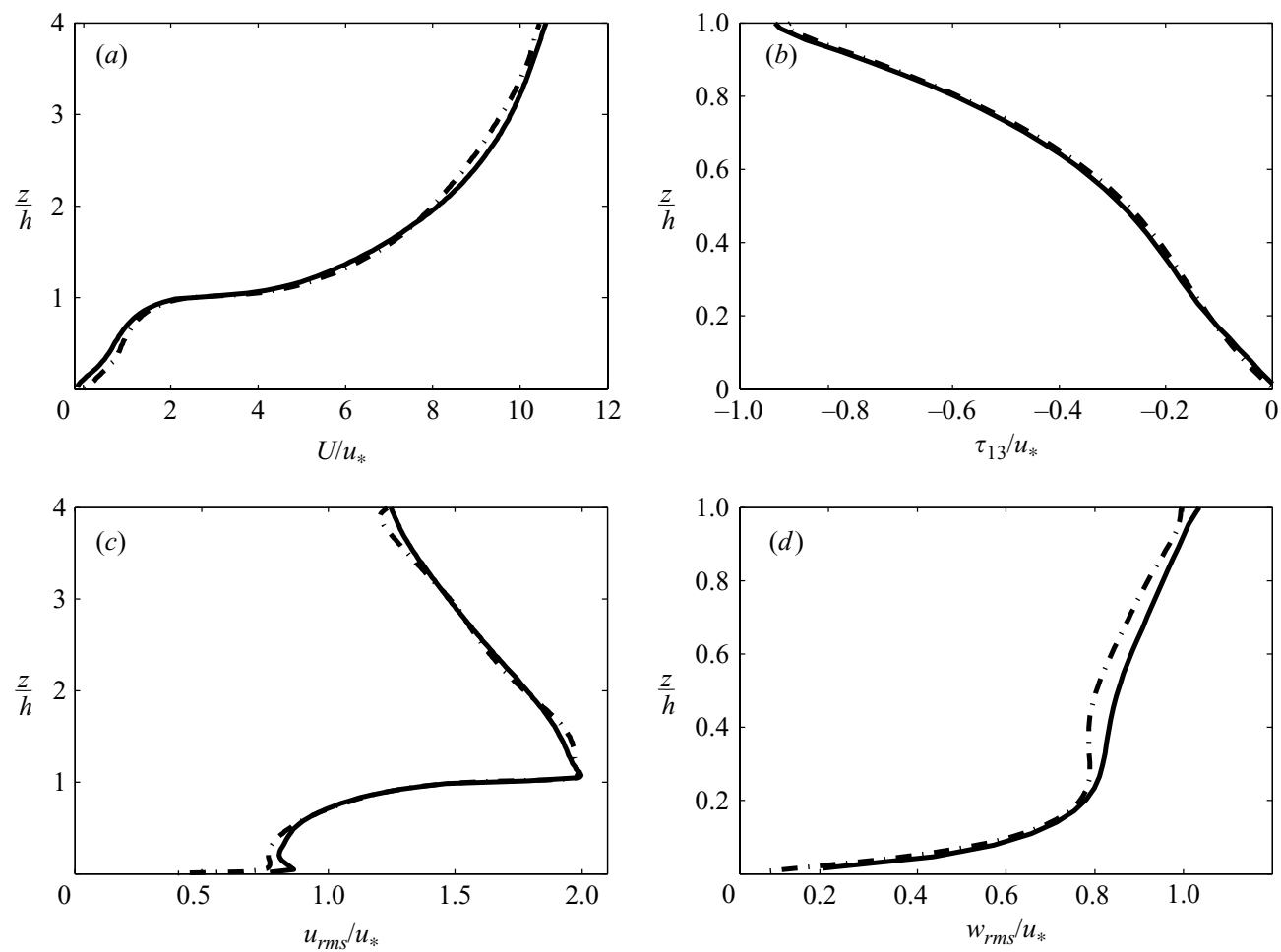

FIGURE 2. Comparison of spatially averaged statistics computed at resolutions of $\Delta=h / 32$ for a domain of dimensions $L=16 h, W=12 h, H=8 h$ (solid lines) and $\Delta=h / 64$ for a smaller domain of dimensions $L=W=H=4 h$ (dot-dashed lines). (a) Mean streamwise velocity, (b) shear stress, $(c)$ streamwise velocity fluctuation, $(d)$ vertical velocity fluctuation.

Instead, a smaller domain with dimensions $L=W=H=4 h$ was used with the aim of comparing results mainly within the array, where the smaller domain size should not have a noticeable effect (Coceal et al. 2006). Figure 2 compares the spatially averaged mean streamwise velocity $U$, the shear stress $\tau_{13}$ and the streamwise and vertical velocity fluctuations $u_{r m s}$ and $w_{r m s}$. These statistics are normalized using the friction velocity $u_{*}$, which is here defined as the value of $\sqrt{\tau_{13} / \rho}$ at the top of the array, where $\rho$ is the fluid density. This is the appropriate velocity scale for rough-bed channel flows, since the total shear stress maximum occurs at the roughness top (see for example Pokrajac et al. 2006).

The agreement between the two runs is in general very good. There are differences in the values of the normal stress root mean square (r.m.s) of up to about $5 \%$, while the differences in the other statistics are in general much smaller. These differences quantify the errors due to the grid resolution (the larger difference in the normal stresses may also be a consequence of the smaller domain height in the higher resolution run, which limits the size of the largest eddies). Since the differences are relatively small, we conclude that the interior of the flow is well resolved. These results are an indication that the turbulence is dominated by large-scale eddies shed from the sharp edges of the cubes, rather than the thin boundary layers on the cube facets. In $\S 3$ the accuracy of the simulations is further demonstrated by detailed comparisons with wind tunnel measurements. 
(a)

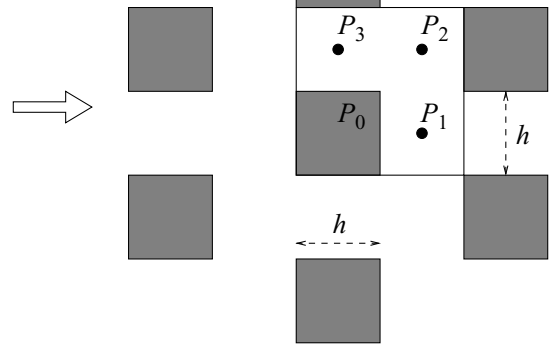

(b)

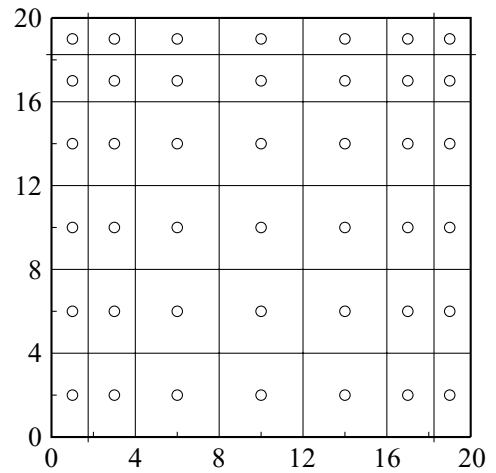

FiguRE 3. Locations of point measurements made by Cheng \& Castro (2002). (a) Velocity measurements at positions indicated by dots. Mean flow is from left to right. (b) Pressure measurements at positions indicated by circles, on front and back faces of a cube. The numbers represent distance in $\mathrm{mm}$.

\section{Flow statistics}

In this section, first- and second-order turbulence statistics computed from the simulations are presented and detailed comparisons are made with measurements performed in the wind tunnel over the same arrangement of cubes by Cheng \& Castro (2002) and Castro et al. (2006). The wind tunnel measurements were made far downstream over an extensive array of cubes of height $h=20 \mathrm{~mm}$, at a fetch where the boundary layer depth was $\approx 7.5 \mathrm{~h}$. Cheng \& Castro (2002) made measurements at Reynolds numbers from 5000 to 12000 (based on free-stream velocity and the cube height) and found that there was little variation over that range of Reynolds numbers. Hence, the published measurements of Cheng \& Castro (2002) and Castro et al. (2006), which were performed at $R e=12000$, are suitable for comparing with the results of the present simulations, which are performed at a Reynolds number of 5800. Pope (2000) points out that the boundary layer flow in a wind tunnel differs from channel flow (as performed in the present DNS) in three principal ways: the boundary layer develops continuously, the wall shear stress is not known a priori, and the outer flow departs from log behaviour. However, Pope notes that in the buffer and $\log$ regions the behaviour is essentially the same as in channel flow, demonstrating this by a remarkable collapse of normalized mean velocity profiles from the boundarylayer experiments of Klebanoff (1954), the boundary-layer DNS of Spalart (1988) and the channel flow DNS of Kim et al. (1987). More recently, Abe, Kawamura \& Matsuo (2001) reported only slight differences in the log region in their channelflow DNS compared with the boundary-layer DNS of Spalart (1988) at a similar Reynolds number. The present comparison of the DNS results with the wind-tunnel data of Cheng \& Castro (2002) and Castro et al. (2006) is further strengthened by the following facts. First, the wind tunnel measurements were performed sufficiently far downstream for the boundary layer development to be negligible in relation to the integral length scales of the flow. Secondly, Cheng \& Castro (2002) had accurate information on the wall shear stress from direct measurements of drag on the cubes. Thirdly, the comparison with their data did not extend beyond the log region.

Figure 3 shows the location of the point measurements performed by Cheng \& Castro (2002) and Castro et al. (2006) in the wind tunnel. Velocity and stress measurements were made at different heights at the horizontal locations denoted 

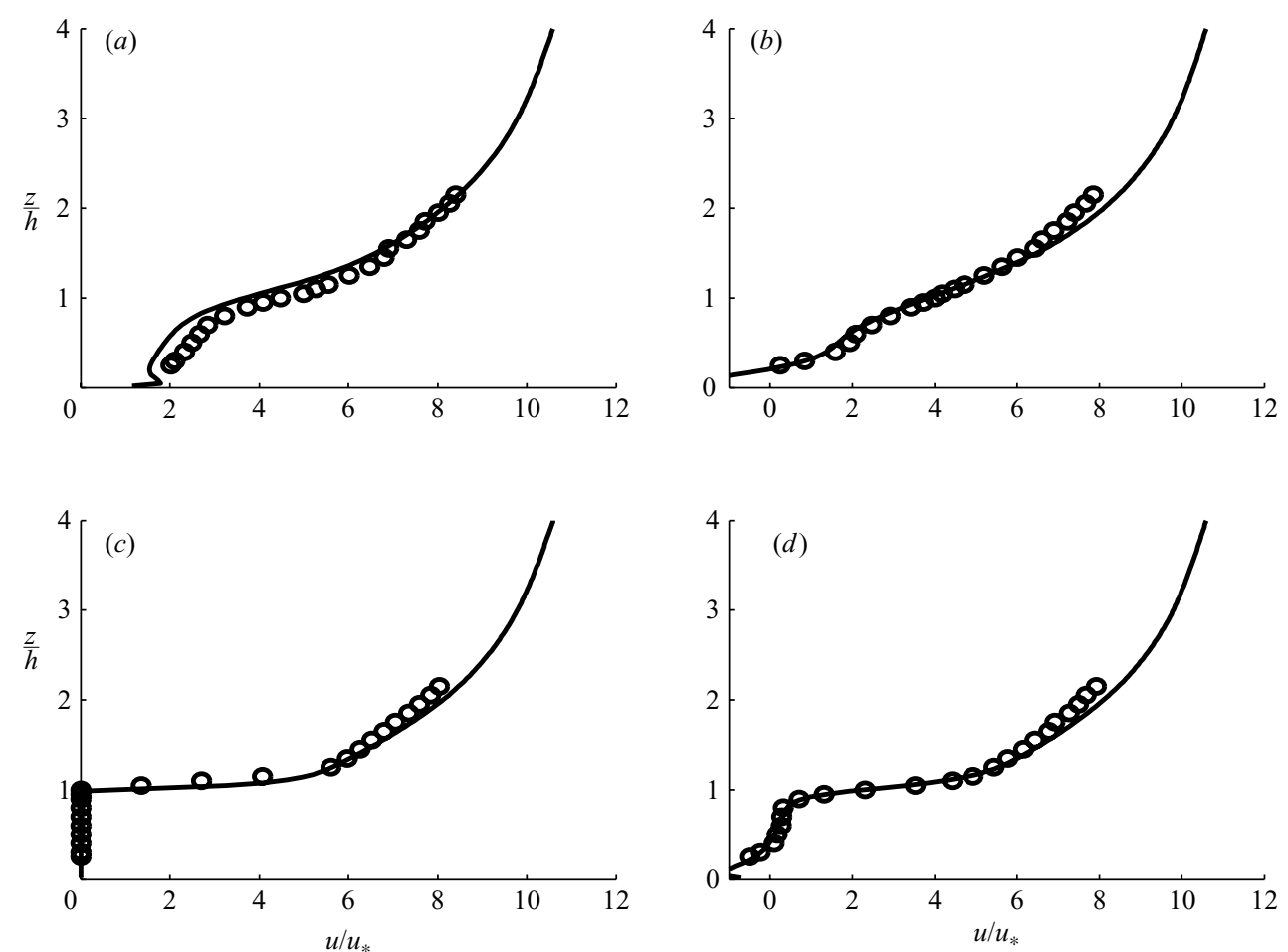

Figure 4. Profiles of mean streamwise velocity $u$ over the four locations indicated in figure 3: $(a)$ in gap, $P_{3} ;(b)$ in front of cube, $P_{2} ;(c)$ on cube, $P_{0} ;(d)$ behind cube, $P_{1}$. Solid lines: computations. Circles: wind-tunnel data from Cheng \& Castro (2002).

by $P_{0}, P_{1}, P_{2}$ and $P_{3}$ in figure $3(a)$. Pressure measurements were made on the front and back faces of a cube by means of pressure tappings at 42 locations on each face as shown in figure $3(b)$.

\subsection{Mean velocity profiles}

The profiles of mean streamwise velocity $u(z)$ at the four locations $P_{0}, P_{1}, P_{2}$ and $P_{3}$ are plotted in figure 4 and are compared to the wind tunnel measurements. The agreement is very good at all four locations. The four profiles are plotted together with that of the spatially averaged velocity $U(z)$ in figure $5(a)$. The four point profiles are very close to the spatially averaged profile almost down to the top of the cubes at $z=h$. There are large differences in the velocity profiles $u(z)$ within the roughness, due to the local flow being much more inhomogeneous there. There is an inflection point in the velocity profile $u(z)$ at the top of the roughness $z=h$ over $P_{1}, P_{2}$ and $P_{3}$ as well as in the spatially averaged velocity profile $U(z)$. The actual values of $\mathrm{d} u / \mathrm{d} z$ at the inflection point varies from point to point and reflects spatial variations in the local shear layer over the top of the cubes.

An inertial sublayer exists above the array where the mean velocity profiles are logarithmic. We follow the meteorological convention and write the log law over a rough surface as (Raupach et al. 1991)

$$
u(z)=\frac{u_{*}}{\kappa} \ln \frac{z-d}{z_{0}},
$$



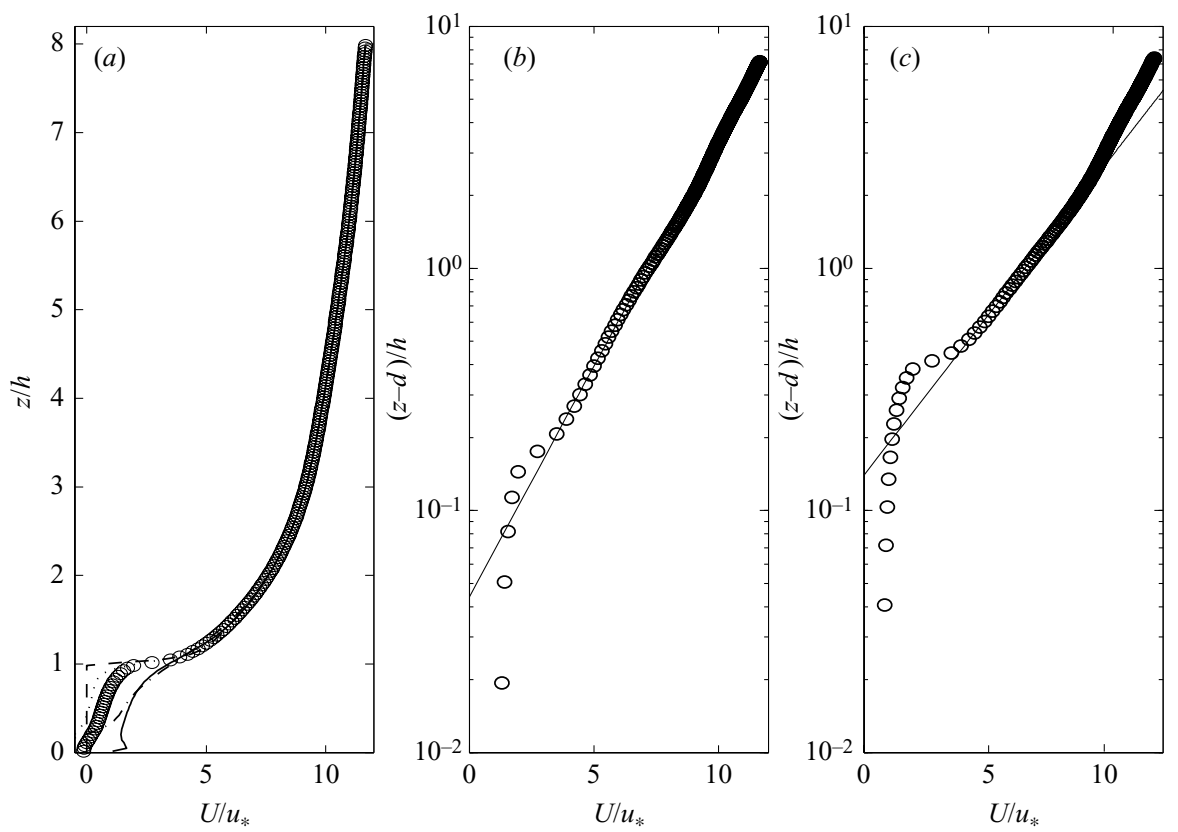

FIGURE 5. Vertical profiles of mean streamwise velocity: (a) plotted vs. $z / h ;(b)$ plotted vs. $(z-d) / h$ on a $\log$ scale, using $\kappa=0.41$ and $d=0.84 h ;(c)$ plotted vs. $(z-d) / h$ on a $\log$ scale, using $d=0.6 h$, as computed using Jackson's method (Jackson 1981). Lines: profiles over positions indicated in figure 3(a). Circles: spatially averaged velocity profiles.

where $\kappa$ is von Kármán's constant, and the parameters $z_{0}$ and $d$ are the roughness length and zero-plane displacement respectively. The roughness length $z_{0}$ is a measure of the capacity of the rough surface to absorb momentum. For fully rough flow, as investigated here, $z_{0}$ depends only on the roughness geometry and is independent of the flow (Raupach et al. 1991). The equivalent sand roughness $k_{s}^{+}$is about 700 , which is well above the fully rough limit of 80 . The physical origin of the zero-plane displacement $d$ is that the flow is displaced upwards, so that $z$ is replaced by $z-d$.

Two different methods are used here to determine $d$. The first is to compute $d$ directly from the mean drag profile within the roughness assuming that $d$ is the mean height of momentum absorption by the surface, based on the experimental results of Thom (1971) on forest-type roughness and subsequent theoretical arguments by Jackson (1981). This yields a method for calculating $d$ as

$$
d=\frac{\int_{0}^{h} z D(z) \mathrm{d} z}{\int_{0}^{h} D(z) \mathrm{d} z},
$$

where $D(z)$ is the drag profile within the array, which can be computed from the pressure difference between the front and back faces of the cubes, as described below. This gives a value for $d / h$ of 0.6 . The second method is to assume the value of von Kármán's constant to be $\kappa=0.41$ and to adjust the value of $d$ to obtain a best fit to the mean velocity profile. This method yields $d / h=0.84$. Almost identical results for $d / h$ were obtained by Cheng $\&$ Castro (2002) using both these methods. 
Figures $5(b)$ and $5(c)$ show the spatially averaged mean streamwise velocity $U(z)$ plotted against $(z-d) / h$ on a semi-log scale using each method. The figure shows that, in both cases, the mean velocity profiles are logarithmic in the range from $z / h=1.1$ to $z / h=2.7$. The extent of this log region in terms of the domain height $H$ is $0.14<z / H<0.34$, and in terms of wall units $50<z^{+}<850$, where $z^{+}=z / \delta_{v}$ with $\delta_{v}=v / u_{\tau}$ and $z^{+}$is measured from the top of the cubes. However, fitting the log profile using the value of $d / h=0.6$ obtained from Jackson's method gives a value for von Kármán's constant of $\kappa=0.31$. This discrepancy in the value of $\kappa$ was also noted by Cheng \& Castro (2002). A similar anomaly was noted by Leonardi et al. (2003) when they applied Jackson's method to compute $d$ to their DNS data over two-dimensional bar roughness at different obstacle densities. They found that the resulting value of $\kappa$ varied between 0.33 and 0.47 . For a frontal area density of 0.25 (which corresponds to that of the present cubical roughness array), they obtained a value for $d / h$ of 0.64 , comparable with the present value of 0.6 ; the slightly higher value in their case is expected because of greater flow displacement over the two-dimensional geometry. The authors of these two papers responded differently to this discrepancy. Cheng \& Castro (2002) concluded that it is likely that Jackson's method does not work for this type of roughness, as opposed to vegetation-type roughness for which there is ample experimental agreement (e.g. Thom 1971). Leonardi et al. (2003) chose to question the outer-wall universality hypothesis, at least at the Reynolds numbers they were investigating. Like these previous authors, we have to leave this as an open issue whose definitive resolution lies outside the scope of the present paper, but that deserves a separate study.

\subsection{Mean pressures}

Figure 6 shows comparisons of computed mean pressure to the pressure tapping measurements performed by Cheng \& Castro (2002). The four plots pertain to different lateral distances from the vertical centreline on the cube face, with the average value being taken from the measurements on each side of the centreline. Each plot shows the vertical profile of the quantity $\Delta p_{n}$ which is the pressure difference between front and back faces of the cube at different locations, normalized by the mean pressure difference. The agreement between the computed and measured values is excellent. The greatest pressure deficit occurs at about $z=0.9 h$ and the minimum at about $z=0.3 h$. The ratio between the maximum and minimum pressure deficit is about 9 . Hence, the aerodynamic pressure drag exerted by the cube on the impinging flow is highly non-uniform.

\subsection{Reynolds stresses and turbulence intensities}

Profiles of Reynolds stress and turbulence intensities normalized by $u_{*}$ at the locations $P_{1}$ and $P_{2}$ are shown in figure 7 and compare well with the wind tunnel measurements of Cheng \& Castro (2002). The Reynolds stress $\overline{u^{\prime} w^{\prime}}$ is in very good agreement with the experimental values both within and above the array. The computed streamwise and vertical turbulence intensities $u_{r m s}$ and $w_{r m s}$ are in good agreement with the measured values. Within the array, the computed values are somewhat lower, with differences of up to about $20 \%$. Note that the Reynolds stresses and turbulence intensities measured at the two locations $P_{1}$ and $P_{2}$ quickly collapse just above the cubes; owing to the uniform height of the cubes the roughness sublayer is relatively shallow.

\subsection{Energy spectra}

The temporal energy spectrum $E_{u u}\left(k z^{\prime}\right)$ of the streamwise velocity component $u$, at a point near the middle of the inertial sublayer $(z=1.7 h)$ is shown in figure $8(a)$ and 

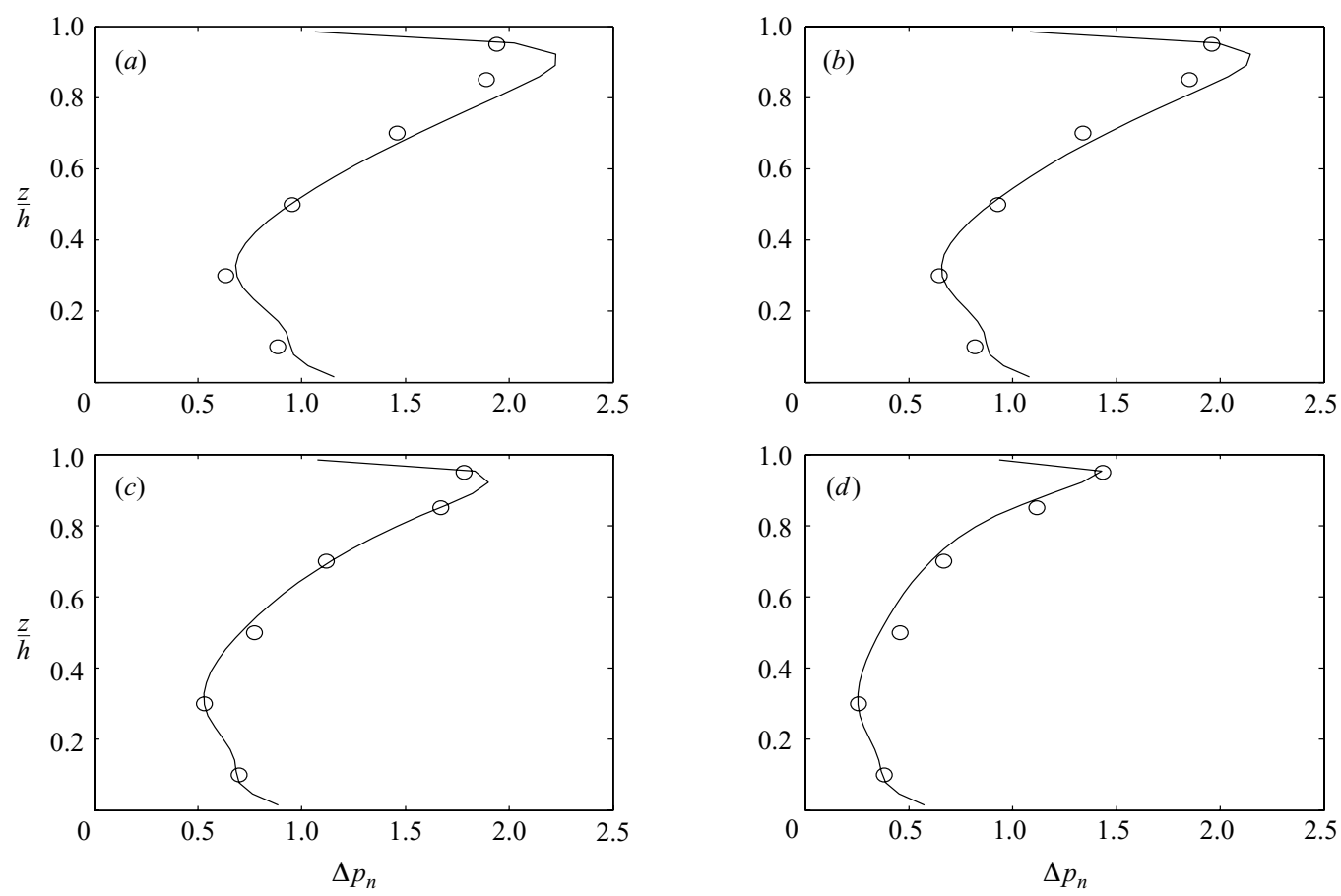

FiguRE 6. Normalized pressure difference $\Delta p_{n}$ between front and back faces of a cube in the array at lateral locations corresponding to the measurement positions indicated in figure $3(b)$. (a) on centreline of cube, $(b) 0.2 \mathrm{~h}$ from centreline, $(c) 0.35 \mathrm{~h}$ from centreline, $(d) 0.45 \mathrm{~h}$ from centreline. Solid lines: computations. Circles: wind-tunnel data from Cheng \& Castro (2002).
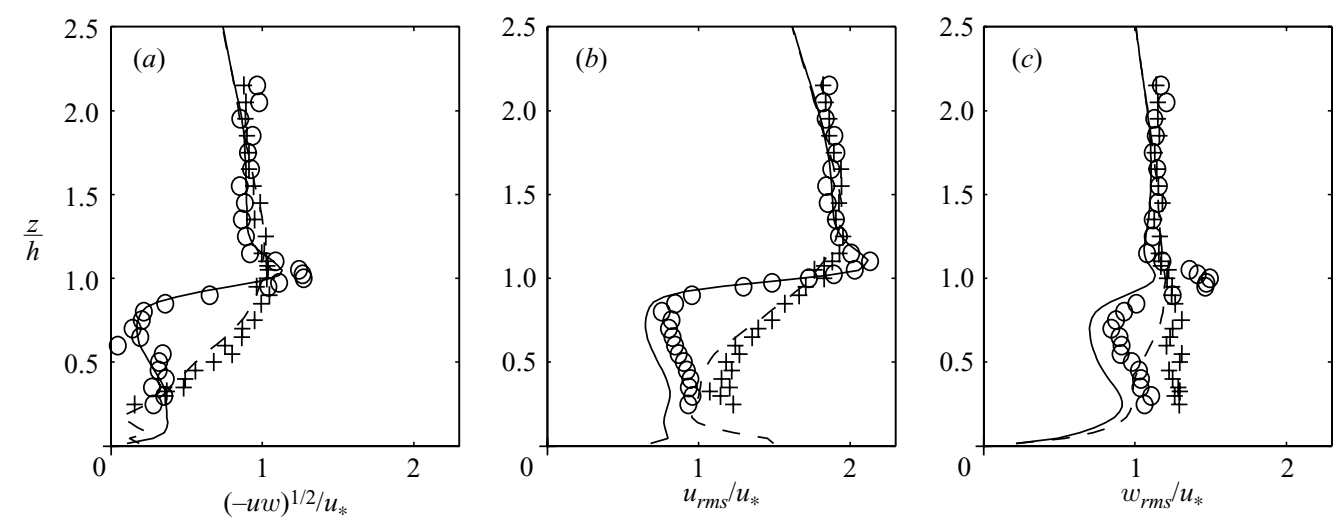

Figure 7. Vertical profiles of $(a)\left(-\overline{u^{\prime} w^{\prime}}\right)^{1 / 2} / u_{*},(b)\left(\overline{u^{\prime 2}}\right)^{1 / 2} / u_{*},(c)\left(\overline{w^{\prime 2}}\right)^{1 / 2} / u_{*}$, in front of cube, $P_{2}$ (dashed lines and plus symbols) and behind cube, $P_{1}$ (solid lines and circles). Lines: computed values. Symbols: wind-tunnel data from Cheng \& Castro (2002).

compared to wind tunnel data at a similar location $P_{2}$. Here $z^{\prime}$ is defined as $z-d$. Similar good agreement has been found for all the locations above the roughness investigated in this paper $(1.2<z / h<4.7)$. Typical 'inner-flow' scaling coordinates are adopted for plotting the energy spectrum, $E_{u u}$, following Perry, Henbest \& Chong (1986). Accordingly, on the $x$-axis $k$ is the streamwise wavenumber defined by $k=2 \pi f / u$, with $f$ being the frequency and $u$ the local mean streamwise velocity. On 

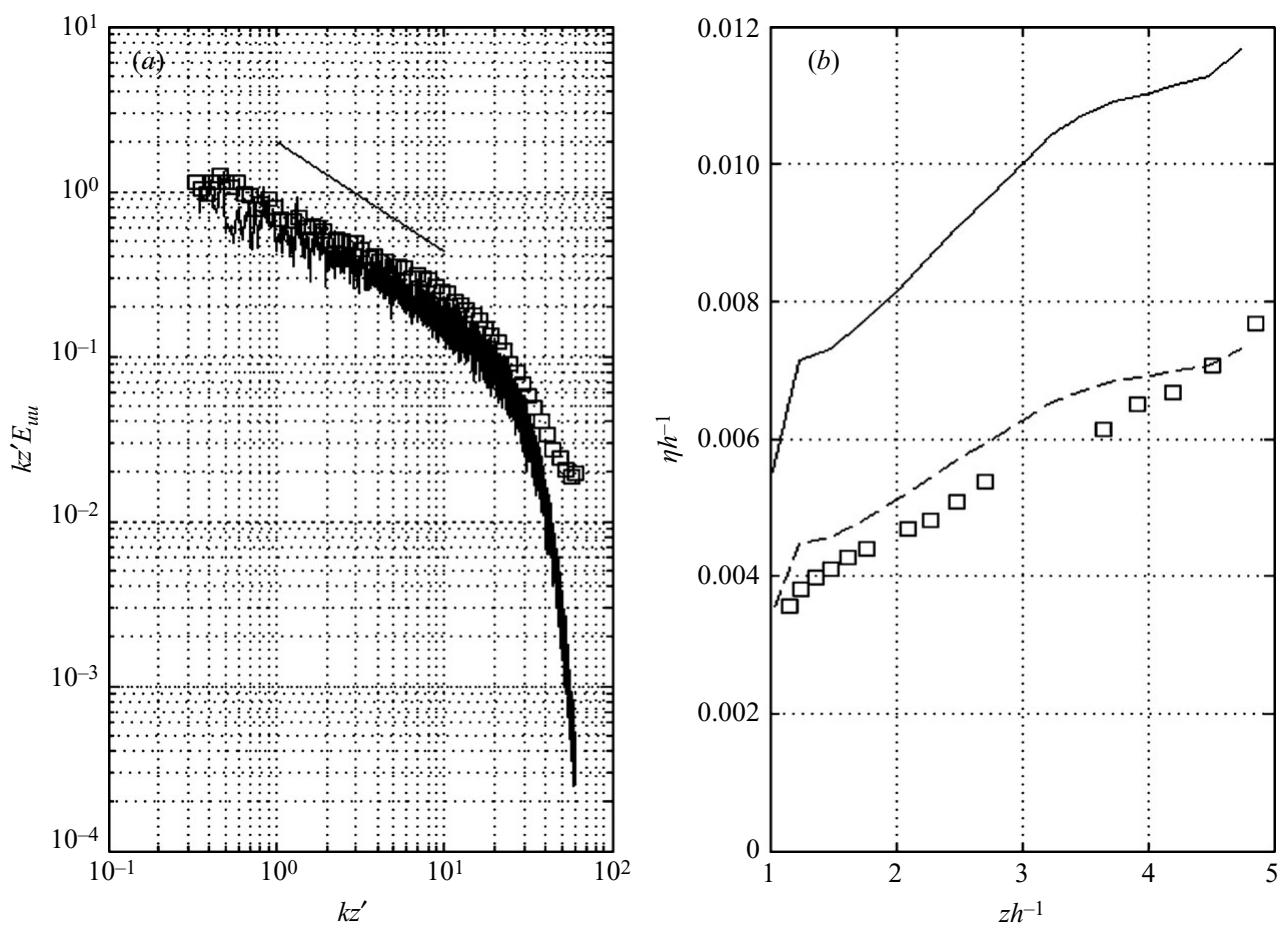

FIGURE 8. (a) Premultiplied energy spectrum $k z^{\prime} E_{u u}$ computed from the simulations (line) and compared with wind-tunnel data (squares) of Castro et al. (2006) at $z=1.7 \mathrm{~h}$. The straight line has a $-2 / 3$ slope. (b) Kolmogorov length scale computed from the simulations (solid line), and rescaled (dashed line) to the Reynolds number of the wind-tunnel experiment of Castro et al. (2006). Squares are values computed from the experimental data.

the $y$-axis, the premultipled spectrum $k z^{\prime} E_{u u}$ is normalized so as to satisfy Parseval's relation:

$$
\int_{0}^{\infty} E_{u u}\left(k z^{\prime}\right) \mathrm{d}\left(k z^{\prime}\right)=\left(u_{r m s} / u_{*}\right)^{2}
$$

with $u_{r m s}$ being the standard deviation of the streamwise velocity component.

From figure $8(a)$ it can be seen that the degree of agreement with the experimental data is remarkably good. The inertial subrange (corresponding to the $-2 / 3$ law) is accurately captured by the numerical results. The differences between the numerical and experimental spectra at wavenumbers $k z^{\prime}>30$ can be attributed to the difference in the Reynolds numbers (Tennekes \& Lumley 1972) between the simulations $(R e=5800)$ and experiment $(R e=12000)$. The fact that there is a ratio of several decades between the numerical energy spectra of the low and high wavenumbers and that there is no evidence of energy pile-up at high wavenumbers give confidence in the simulation accuracy.

Figure $8(b)$ shows the variation of the Kolmogorov scale $\eta=\left(v^{3} / \epsilon\right)^{1 / 4}$ with height. The dissipation rate $\epsilon$ was estimated by fitting a $k^{-5 / 3}$ function to the energy spectrum over the inertial-scale range, using the Kolmogorov approximation $E(k)=C \epsilon^{2 / 3} k^{-5 / 3}$, with $C=0.47$. Note that a similar procedure and coefficient is used in Castro et al. (2006), making the comparison with the experiment consistent. The inertial-scale range is approximated as being the region where the dissipation spectrum is flat. The dashed line in figure $8(b)$ represents a rescaling that takes into account the Reynolds 

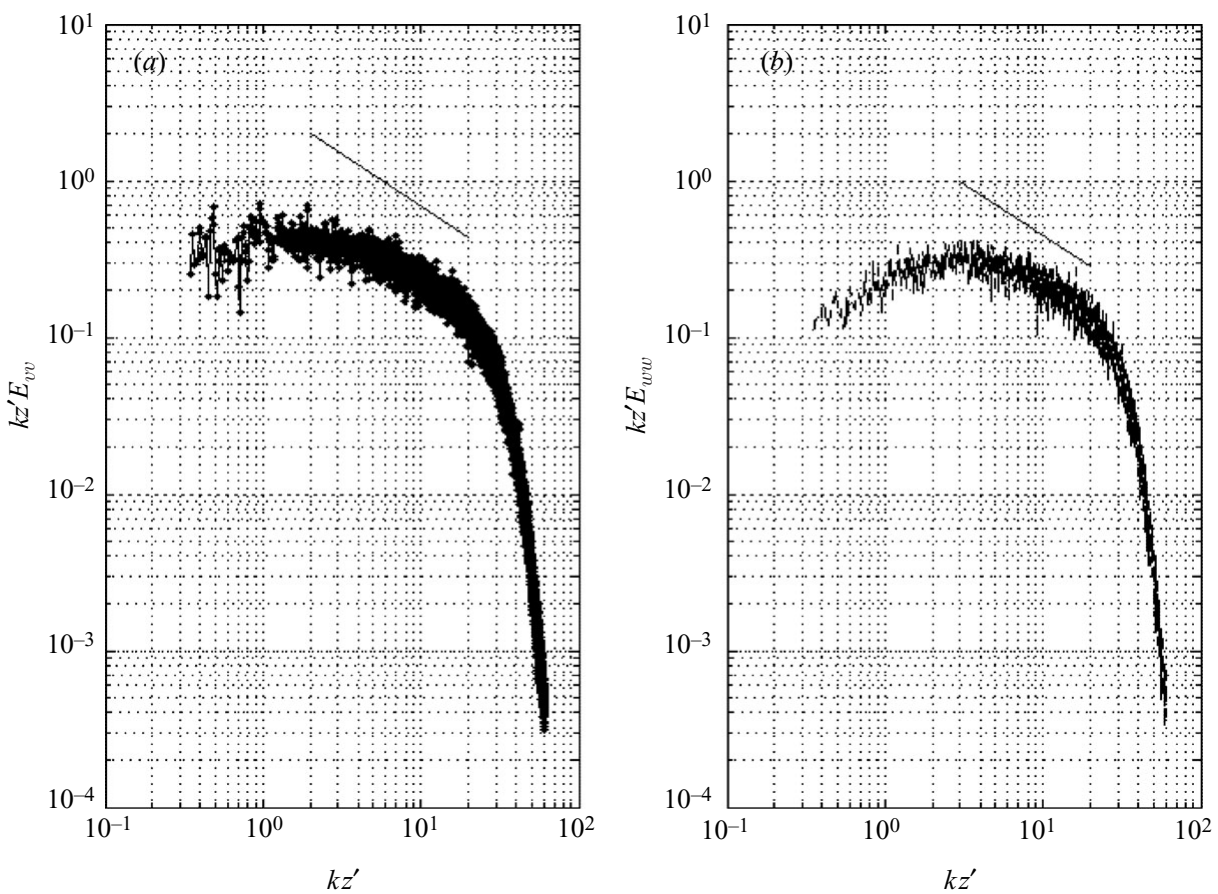

FIgURE 9. Premultiplied energy spectra $k z^{\prime} E_{v v}$ and $k z^{\prime} E_{w w}$. The straight lines have a $-2 / 3$ slope.

number difference between the experiments and numerical simulation. The collapse of the two curves is good. This indicates on one hand that the smallest scales in the flow are simulated properly and on the other hand that the similarity assumption between the two Reynolds numbers is correct, in agreement with Cheng \& Castro (2002).

Figure 9 shows the corresponding premultiplied spectra of the spanwise and wallnormal components, $k z^{\prime} E_{v v}$ and $k z^{\prime} E_{w w}$. As for their streamwise counterpart $k z^{\prime} E_{u u}$, there is no energy pile-up at high wavenumbers and the $-2 / 3$ law is well captured.

\subsection{Two-point correlations and structure angles}

Two-point velocity correlations, defined by

$$
R_{u u}(r, \delta r)=\frac{\overline{u^{\prime}(r) u^{\prime}(r+\delta r)}}{\sqrt{\overline{u^{\prime 2}(r)}} \sqrt{\overline{u^{\prime 2}(r+\delta r)}}},
$$

are computed for separations $\delta r$ in the streamwise, lateral and vertical directions using a fixed reference at $z=1.7 \mathrm{~h}$. These compare well with the wind-tunnel data of Castro et al. (2006) in figure 10. We note that $R_{u u}(\delta x)$ has a value of 0.15 for a streamwise separation $\delta x$ of half the domain size. Hence, even though the present domain length is more than five times larger than the integral length scale $L_{x} \approx 3 h$ deduced by Castro et al. (2006) from wind-tunnel measurements over a much longer distance (see $\S 2$ ), or more than 2.5 times larger than the eddy separation length scale $2 \pi L_{z} \approx 6 h$ (Raupach et al. 1991), the two-point correlation does not quite decay to zero in half the domain length. It would therefore be desirable to have a larger domain in future simulations. We also note, however, that the computed values of 


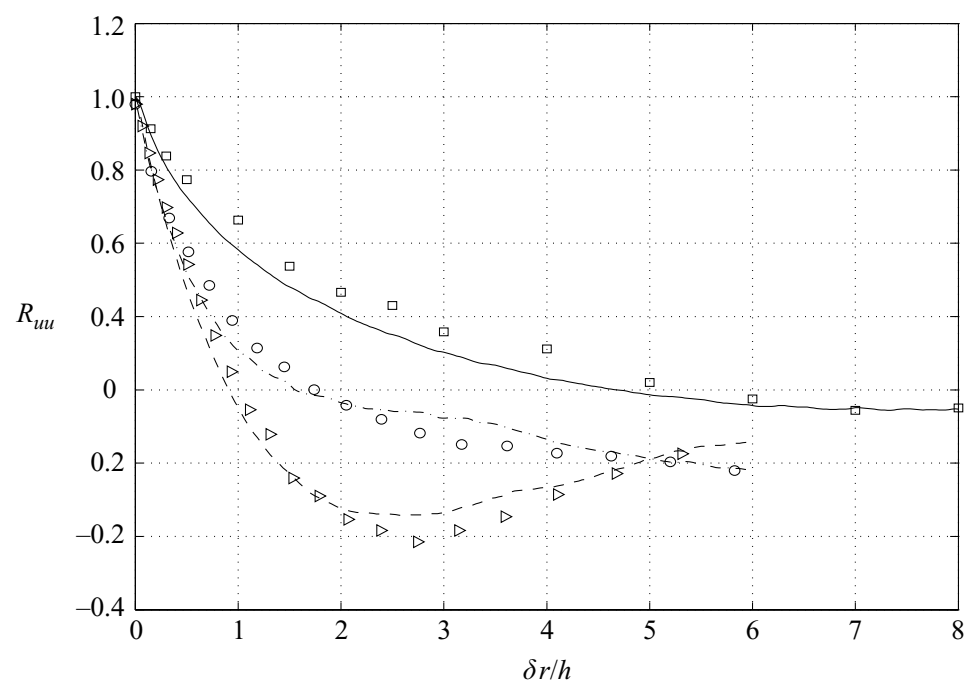

FIGURE 10. Two-point correlations $R_{u u}(\delta r)$ computed for separations in the streamwise (solid line and squares), lateral (dashed line and triangles) and vertical (dot-dashed line and circles) directions. Lines: computed from simulations. Symbols: computed from wind-tunnel data of Castro et al. (2006).

$R_{u u}(\delta x)$ for large streamwise separations are almost equal to the wind-tunnel data. Hence, in practice the errors due to the limited domain appear to be small.

The two-point correlations $R_{u u}(r)$ are also computed in the whole vertical $(x, z)$ plane for a given reference location relative to a cube. To obtain smoother plots, timeseries data from three different vertical slices at $y=2.5 h, 5.5 h$ and $8.5 h$ (referring to figure 1) are used. In addition, reference locations at a streamwise distance of $0.5 \mathrm{~h}$ upstream of each cube are selected in each slice. This gives a total of 12 realizations from which the two-point correlations are computed and ensembleaveraged. Figure 11 shows contour plots of $R_{u u}$ for different vertical locations of the reference point throughout the $\log$ region starting from $z=1.25 \mathrm{~h}$ up to $z=2.75 \mathrm{~h}$ for every $0.5 \mathrm{~h}$. Note that, in computing the ensemble averages, the coordinate system has been shifted so that the maximum correlation is in the middle of the domain, at $x=8 h$. The contours are elongated in the streamwise direction and are inclined at a mean angle that depends on the height of the reference point. To quantify the size and inclination angle of these structures, the locus of the maxima in the correlation is plotted for values of $R_{u и}$ from 1.0 to 0.3 (following Krogstad \& Antonia 1994, the 0.3 correlation level was chosen to ensure sufficiently accurate correlations). The length of the structure, as defined by the $R_{u u}=0.3$ contour, increases monotonically with height.

Figure 12 shows that the mean inclination angle decreases sharply with height from $21.6^{\circ}$ at $z=1.0 \mathrm{~h}$ to $14.4^{\circ}$ at $z=1.5 \mathrm{~h}$, thereafter decreasing more slowly to a value of $12.2^{\circ}$ at $z=2.75 \mathrm{~h}$. These results are consistent with measurements performed by Castro et al. (2006), who observed that the inclination angle decreased from $18.4^{\circ}$ to $10.3^{\circ}$ from $z=1.0 \mathrm{~h}$ to $z=2.75 \mathrm{~h}$, with what appears to be scatter between. As pointed out by these authors, this behaviour is different to what is observed over smooth walls (Adrian et al. 2000; Marusic 2001) where the structure angle is observed to increase monotonically with increasing distance from the wall. Earlier, Krogstad \& Antonia (1994) computed two-point correlations in the same plane for a smooth wall 
(a)

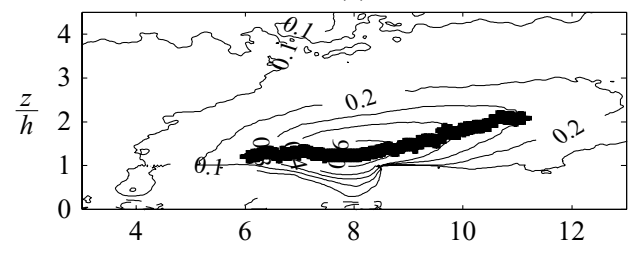

(c)

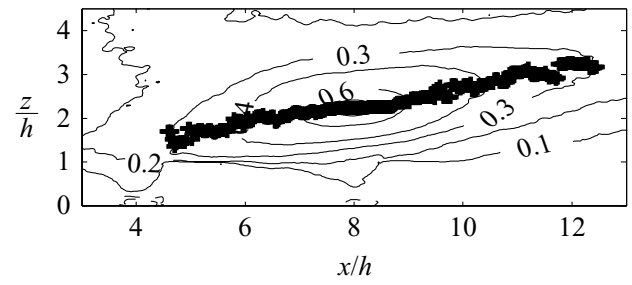

(b)

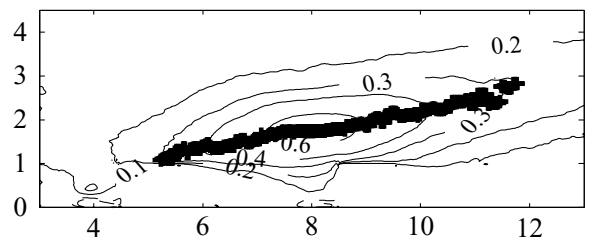

$(d)$

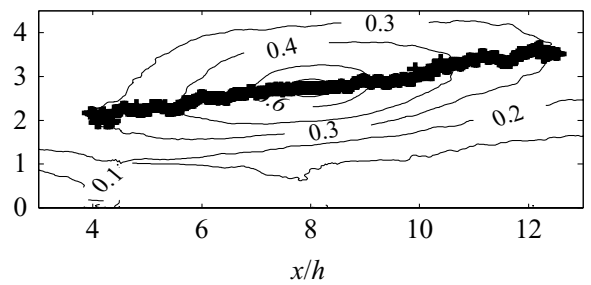

FIGURE 11. Two-point correlations $R_{u u}$ computed and ensemble-averaged in the $(x, z)$-planes through $y=2.5 h, 5.5 h$ and $8.5 h$ and with reference points located $0.5 h$ upstream of a cube. Plots correspond to vertical locations of the reference point of $(a) z=1.25 h,(b) 1.75 h$, (c) $2.25 \mathrm{~h}$ and $(d) 2.75 \mathrm{~h}$ respectively. The coordinate system has been shifted so that the maximum correlation is in the middle of the domain, at $x=8 h$. Only part of the domain is shown for greater clarity. Also plotted is the locus of the maximum correlation.

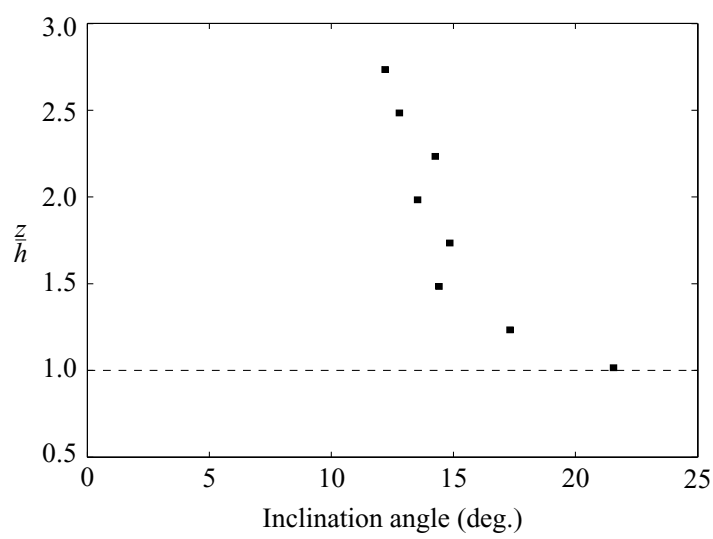

FIGURE 12. Average inclination angle of the locus of maximum correlation, deduced from figure 11. See text for explanation.

and a rough wall consisting of a steel wire mesh. They found that the inclination of the $R_{u u}=0.3$ contour had an average value of about $10^{\circ}$ for the smooth wall and a much larger value of about $38^{\circ}$ for the rough wall. They attributed this large difference partly to larger wall-normal velocity fluctuations over the rough wall, which is associated with reduced damping close to the rough surface.

In anticipation of the results shown in $\S 4$, an alternative (though related) account may be given in terms of the dominant coherent structures in the flow, which are in fact hairpin vortices. In a shear flow, vorticity is rotated towards the streamwise direction by the antisymmetric part of the shear tensor $\mathrm{d} U_{i} / \mathrm{d} x_{j}$ and stretched by the symmetric part. At the same time, self-induction due to the legs of the hairpin 
vortex tends to rotate the vortex towards the vertical. Therefore, over a smooth wall, the angle of inclination of a hairpin vortex is determined by the equilibrium between this self-induction and the mean shear. Near the wall, the mean shear is largest; also the self-induction is reduced due to the effect of an image vortex. Both these effects decrease with distance from the wall. One must also take into account the fact that the self-induction of larger hairpins further away from the wall is smaller than that of smaller hairpins close to the wall (Adrian et al. 2000). Over a smooth wall, it is observed that the inclination angle of hairpin vortices increases monotonically with distance from the wall (Adrian et al. 2000; Marusic 2001). Hence, it appears that the combined decrease of the mean shear and the image vortex effect more than offsets the decrease of the self-induction with increasing wall distance. Near a rough wall the image vortex is much further away if the reference location is over a cavity, as in the present case. Induction due to the vortex legs is then stronger than over smooth walls, and the inclination angle of the vortex is correspondingly larger near the wall. Given this weaker image vortex, it is then possible that the decrease of the inclination angle with wall-normal distance is here associated with a more rapid decrease of the self-induction of the (larger) hairpin vortices compared with the decrease in the mean shear. This would be an interesting subject for a more quantitative future investigation.

\subsection{Quadrant analysis}

In the quadrant analysis technique (Wallace, Eckelman \& Brodkey 1972) one considers the frequency of occurrence and contribution to Reynolds stress $\overline{u^{\prime} w^{\prime}}$ of velocity fluctuations lying in the four quadrants defined by

$$
\left.\begin{array}{l}
\text { Q1 }: u^{\prime}>0, w^{\prime}>0, \\
\text { Q2 : } u^{\prime}<0, w^{\prime}>0, \\
\text { Q3 : } u^{\prime}<0, w^{\prime}<0, \\
\text { Q4 : } u^{\prime}>0, w^{\prime}<0 .
\end{array}\right\}
$$

Q1 and Q3 events are generally rare and contribute little to the Reynolds stress. The more significant events are Q2 (ejections) which transport low-momentum fluid upwards, and Q4 events (sweeps) which transport high-momentum fluid downwards. The relative frequency of occurrence of ejections and sweeps and their contribution to $\overline{u^{\prime} w^{\prime}}$ is an indicator of the turbulence structure. Boundary layers for example are characterized by a greater number of sweeps relative to ejections, but the fewer ejections contribute more to $\overline{u^{\prime} w^{\prime}}$ because they tend to be stronger The opposite is true in mixing layers, where fewer but more energetic sweeps dominate the contribution to $\overline{u^{\prime} w^{\prime}}$ (Finnigan 2000).

Castro et al. (2006) computed contributions from Q2 and Q4 events based on measurements at $P_{0}, P_{1}, P_{2}$ and $P_{3}$ and showed there were significant variations with probe location close to and within the array. Here statistics are summed over all points in the domain to avoid this problem. About 1000 snapshots sampled every $0.1 T$ were used and the results represent a sum over all points in the $(x, y)$-plane. This gave a total of 200 million events for each value of $z$. Figure 13 shows the resulting profiles of the relative number of each type of event and the relative magnitude of their contribution to $\overline{u^{\prime} w^{\prime}}$, expressed as a fraction of the sum of the magnitudes in all the quadrants.

Figure 14(a) shows that the relative number of ejections and sweeps, and their relative contribution to the Reynolds stress, are both relatively smooth functions of height. Away from the wall, there are more sweeps but the ejections are more 

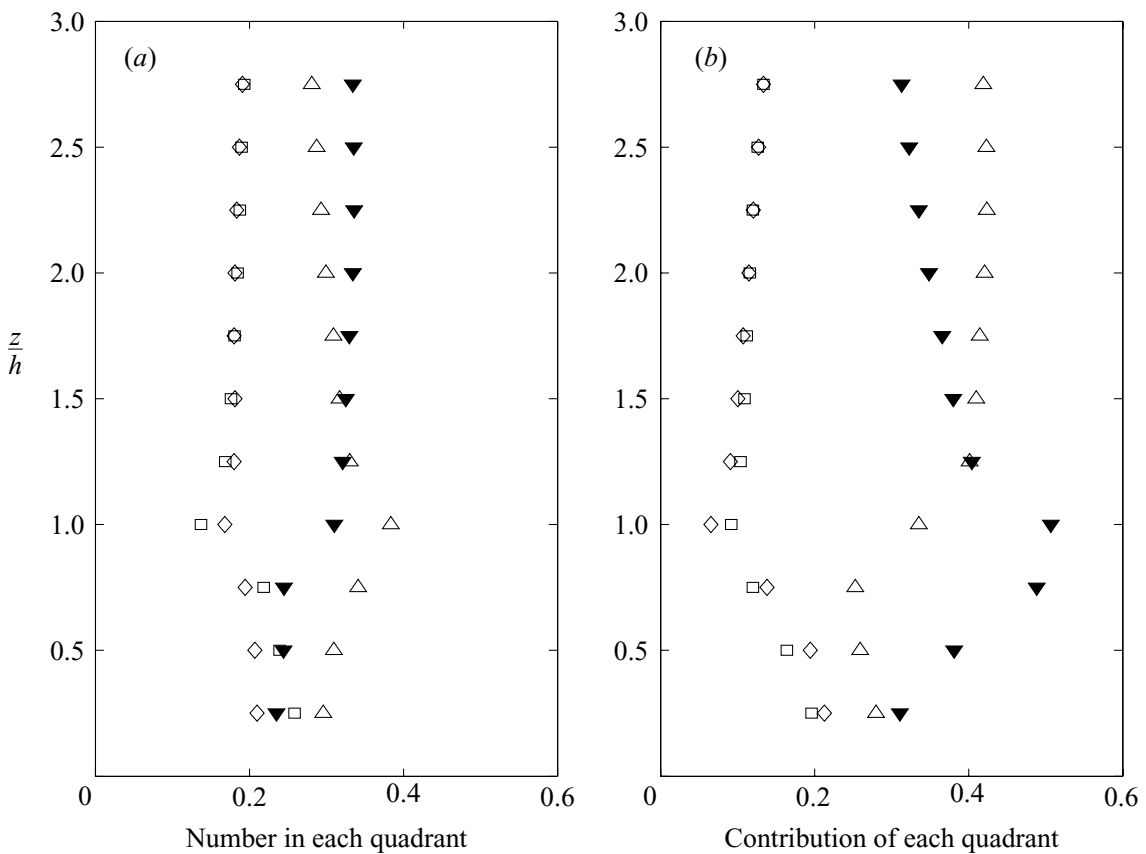

FIGURE 13. (a) Relative number of events in each quadrant as a function of height. (b) Relative contribution to $\overline{u^{\prime} w^{\prime}}$ of events in each quadrant as a function of height. Squares: Q1; triangles up: Q2; diamonds: Q3; filled triangles down: Q4.
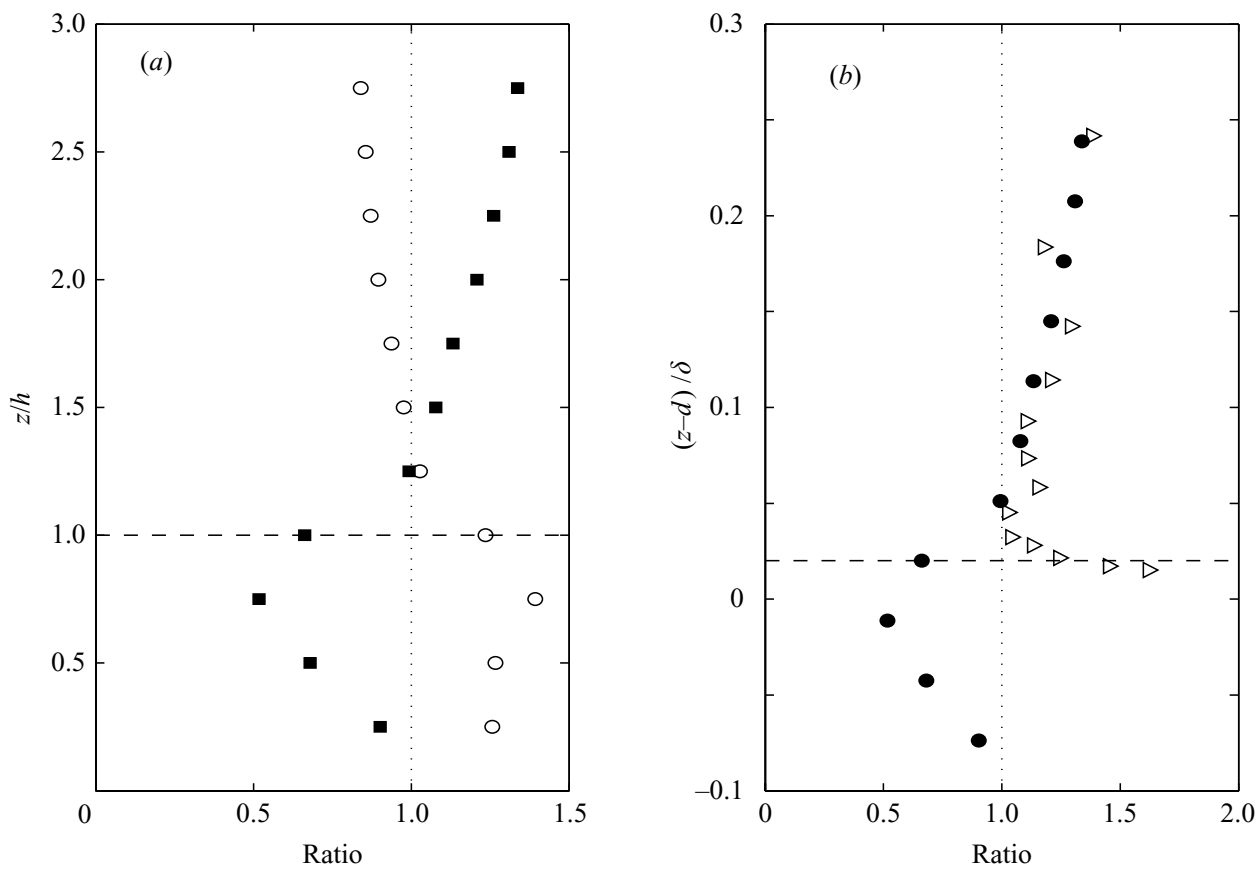

FigURE 14. (a) Ratio of number (circles) and contribution to $\overline{u^{\prime} w^{\prime}}$ (squares) of Q2 events to that of Q4 events from the DNS data, as a function of height. (b) Ratio of contribution to $\overline{u^{\prime} w^{\prime}}$ (circles) of Q2 events to that of Q4 events as a function of height for the DNS data, plotted as a function of $(z-d) / \delta$ with $d=0.84 h$; smooth wall data from Krogstad \& Antonia (1994) (triangles). Dashed lines indicate top of roughness. 
significant based on their contribution to the Reynolds stress. However, close to the top of the obstacles, this pattern is reversed, and contributions to the shear stress come mainly from fewer but more energetic Q4 events. These results agree with the conclusions of Raupach (1981), who observed that Q4 contributions account for most of the stress close to rough surfaces. The crossover point between sweep dominance and ejection dominance is at around $z=1.25 h$, where Q2 and Q4 events are equally probable and contribute equally to the stress. Within the array, the ratio of number of ejections to that of sweeps reaches a maximum, and the ratio of their corresponding contributions to $\overline{u^{\prime} w^{\prime}}$ attains a minimum, at $z=0.75 \mathrm{~h}$.

In figure $14(b)$, the fractional contribution of ejections to sweeps is compared with smooth-wall data from Krogstad \& Antonia (1994). The data are plotted in outer units, with the wall distance origin shifted by the displacement height $d$ in the rough-wall case. The value of $d$ is here taken to be $0.84 h$ (see $\S 3.1$ ), but a similar comparison results with $d=0.6 h$. The plot shows a good collapse of the ratio from about $0.1 \delta$ above the effective origin of the two surfaces, but a rapidly increasing difference close to the surfaces, from about $0.05 \delta$ downwards. The minimum ratio for the smooth wall is just over 1 , so that contributions from sweeps never dominate ejections, and the ratio increases rapidly to more than 1.5 close to the smooth surface. By contrast, the corresponding ratio for the present roughness is about 0.6 at the effective origin $z=d$ of the rough surface. Krogstad, Antonia \& Browne (1992) also observed stronger sweeps near roughness relative to smooth walls. They interpreted this as a consequence of reduced damping due to the open nature of the roughness. Krogstad \& Antonia (1999) found that in the case of two-dimensional bar roughness ejections are much reduced and Krogstad et al. (2005) proposed that this may be due to low-momentum fluid being trapped between the roughness elements.

A different view is given by Raupach, Finnigan \& Brunet (1996) and Finnigan (2000) regarding similar observations of stronger sweeps over vegetation canopies. This view is based on an analogy with the instability modes of the plane mixing layer (Rogers \& Moser 1992). According to this model, a primary inviscid (Rayleigh) instability is generated by the inflected velocity profile near the top of the roughness canopy. This instability gives rise to large, rapidly developing transverse vortices (rollers). Secondary instabilities in the rollers then produce streamwise counter-rotating vortices (ribs) of the same scale with a preferential pairing that gives rise to strong sweeps between the ribs. This mixing layer analogy is supported by the close resemblance of a number of statistics over vegetation canopies with the plane mixing layer (Finnigan 2000), and by detailed analysis of wind-tunnel data using proper orthogonal decomposition (Finnigan \& Shaw 2000). In the present case, we also have a strongly enhanced ratio of the Q4 vs. Q2 contribution near the top and within the array, as well as more localized inflected velocity profiles (figure 5). It is therefore possible that a similar mechanism to that over vegetation canopies may operate near the top of the present roughness.

\section{Coherent structures}

Both the results based on two-point correlations and those based on quadrant analysis provide evidence of organized motions in the flow. In this section we explicitly demonstrate the existence of coherent structures in the flow over the present roughness, and compute some of their statistical properties.

\subsection{Instantaneous flow visualization}

Flow visualization has been an important tool in the investigation of coherent structures since the earliest experimental studies (Brown \& Roshko 1974). Numerical 
(a)

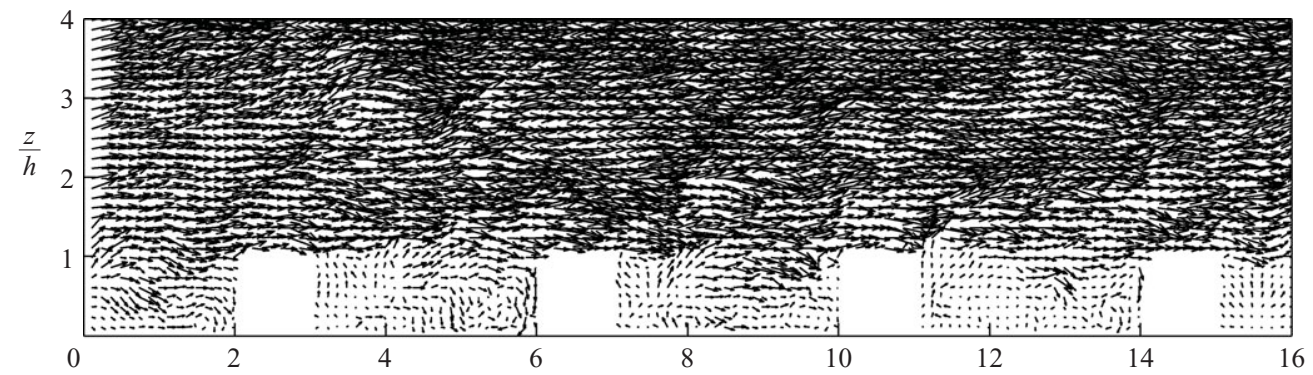

(b)

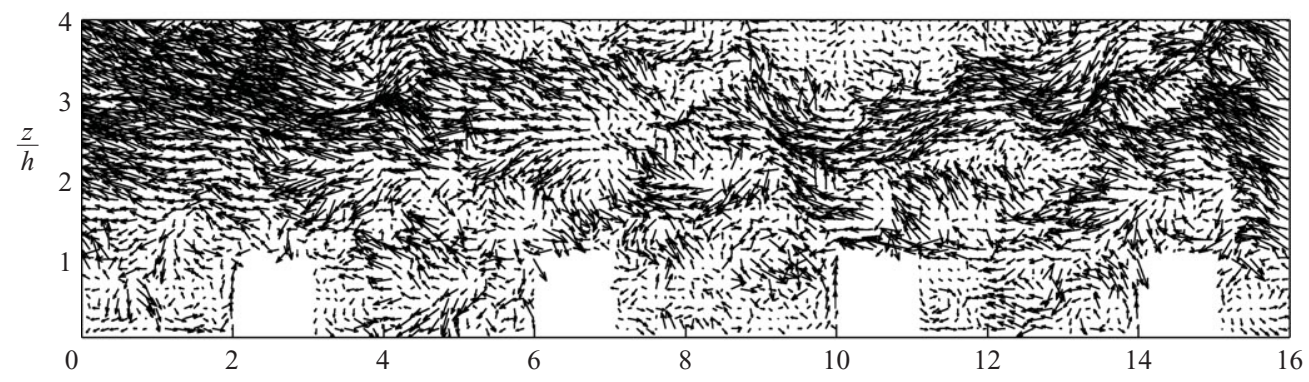

(c)

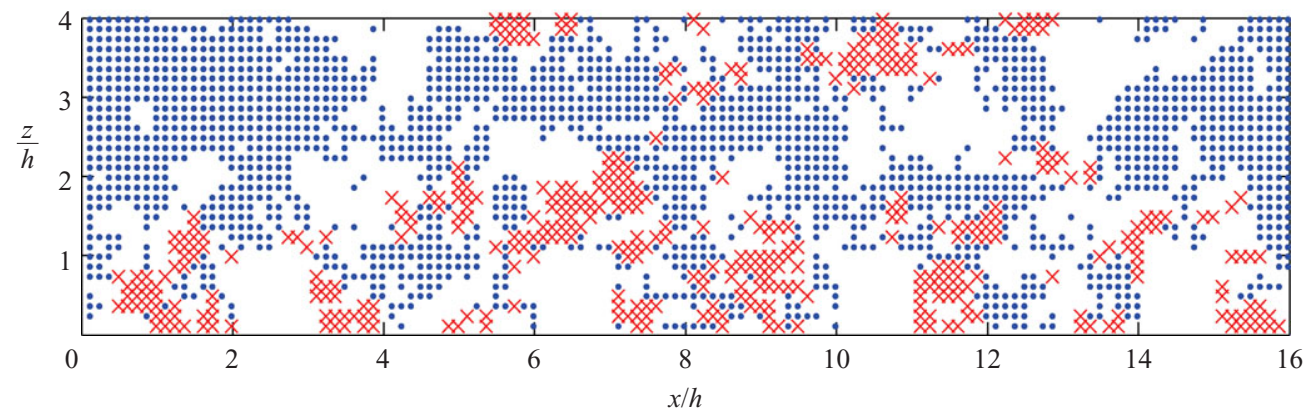

FIGURE 15. (a) An instantaneous wind-vector plot of $(u, w)$ in an $(x, z)$-plane through the middle of the cubes shown. The vectors are plotted at reduced resolution for clarity. (b) Corresponding wind-vector plot of the fluctuating velocity components $\left(u^{\prime}, w^{\prime}\right)$ in the same plane. (c) Spatial distribution of Q2 and Q4 events for the same snapshot. Blue dots: Q2 events. Red crosses: Q4 events.

computations like the present one allow detailed visualizations of three-dimensional flow structures, and it is useful to begin by studying some instantaneous snapshots of the flow. Phenomenologically, we focus on three visually identifiable types of structures, which are all related: spatially organized Q2 and Q4 events, low-momentum regions (LMRs) and vortical structures (hairpin vortices).

\subsubsection{Spatial organization of $Q^{2}$ and $Q 4$ events}

It was shown in $\S 3$ that Q2 and Q4 events are responsible for most of the vertical transport of momentum. But how are these events distributed in space? Figure 15(a) shows an instantaneous snapshot of velocity vectors $(u, w)$ in the streamwise-vertical $(x, z)$ plane through the middle of a cube while figure $15(b)$ shows the corresponding wind-vector plot of the fluctuating velocity components $\left(u^{\prime}, w^{\prime}\right)$. Figure $15(c)$ plots the locations of Q2 and Q4 events in the same snapshot as blue dots and red crosses 
(a)

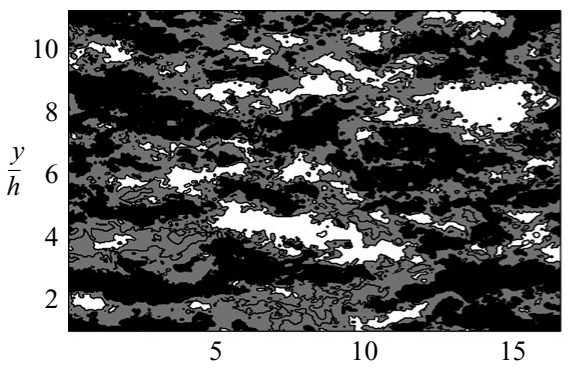

(c)

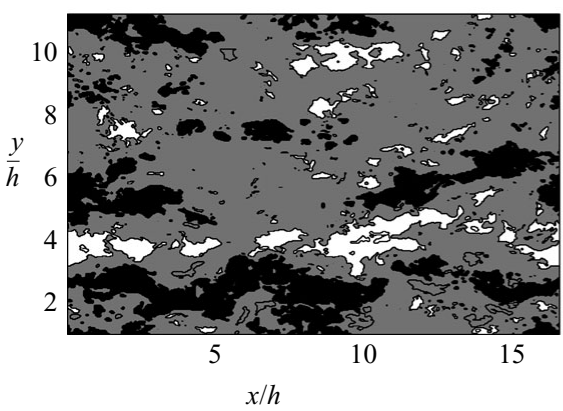

(b)

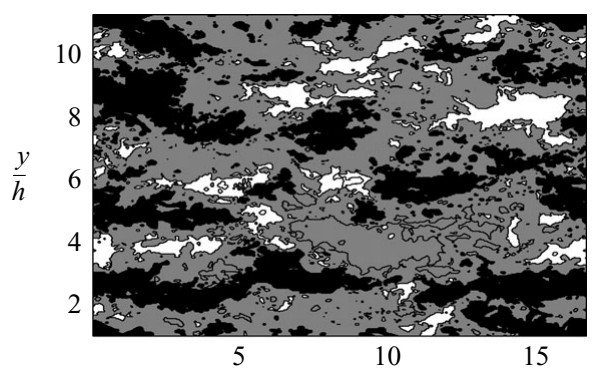

(d)

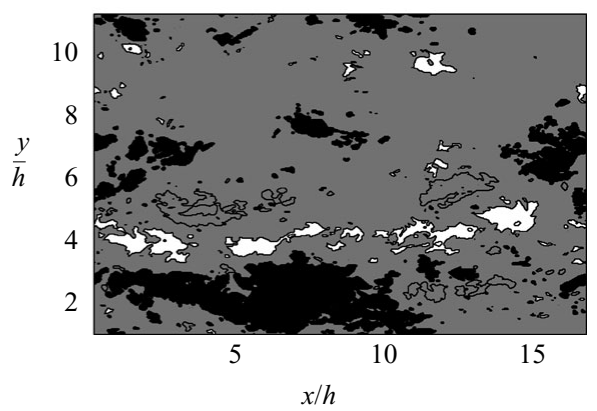

FIGURE 16. Instantaneous snapshot showing contour plots of streamwise velocity at different heights: $(a) z=1.25 h,(b) z=1.5 h,(c) z=2.0 h,(d) z=2.5 h$. Black regions: $u<0.8 u_{m}$. White regions: $u>1.2 u_{m}$. Here $u_{m}$ is the local mean velocity.

respectively. The most striking feature of this plot is that the Q2 and Q4 events are not randomly distributed in space, but are grouped into distinct regions that span a range of scales. The existence of these patterns is much more evident than in the wind vector plots. Such patterns are observed in every snapshot, and a time sequence of such snapshots shows that they advect and evolve coherently.

\subsubsection{Low-momentum regions}

Another, equally ubiquitous, type of large-scale structure is a low-momentum region (LMR), defined as a region in the flow where the instantaneous streamwise velocity is lower than the local mean by some specified threshold. Elongated LMRs have been found to be very widespread in the log region over smooth walls (Tomkins \& Adrian 2003). They are much larger than the better known near-wall streaks (Kline et al. 1967), and can be about 500 wall units wide or more. Figure 16 shows a contour plot of fluctuating streamwise velocity $u^{\prime}$ for a snapshot at different heights above the present cube roughness. The black regions correspond to $u^{\prime}$ being more than $20 \%$ lower than the local mean while the white regions correspond to $u^{\prime}$ being more than $20 \%$ higher. The LMRs (black) are elongated in the streamwise direction, similar to those observed by Tomkins \& Adrian (2003). The LMRs are more numerous near the top of the cubes, and become less so with increasing distance above the cubes until they disappear above about $z=3 h$, outside the log region. Both the average thickness and the average length of the LMRs increase with height. Like the spatial patterns of Q2 and Q4 events, the LMRs are present in almost every snapshot. An animated 
sequence of snapshots shows that the LMRs tend to persist for long periods, typically tens of eddy turnover times, and moving downstream with the fluid. They then break up quasi-randomly and re-form elsewhere following a period of disorganization.

\subsubsection{Vortical structures}

Adrian et al. (2000) and Tomkins \& Adrian (2003) present detailed observational evidence from PIV measurements in the log region over smooth walls that the LMRs are associated with coherent groups, or packets, of hairpin vortices. Vortical hairpin-like structures also exist in the present flow. Vortex cores can be directly visualized as negative isosurfaces of $\lambda_{2}$, the second eigenvalue of the tensor $\boldsymbol{S}^{2}+\boldsymbol{\Omega}^{2}$, where $\boldsymbol{S}$ and $\boldsymbol{\Omega}$ are the symmetric and antisymmetric parts of the velocity gradient tensor $\nabla \boldsymbol{u}$ (Jeong \& Hussain 1995). Figure 17 shows such a visualization for one snapshot. It shows a large number of vortices around LMRs (here visualized in three dimensions). However, because the Reynolds number is high there is interaction amongst many scales and the individual shapes and organization of the vortices are not very clear. A filtering method must be used to isolate vortices at a particular scale. One method is described in the next subsection below. Alternatively, a clearer picture is obtained at lower Reynolds numbers, as in figure 18, in which the Reynolds number is $R e=1200$ or $R e_{\tau}=125$. While still being in the fully rough regime (see e.g. Djenidi et al. 1999) there is now less inter-scale interaction so that the flow structure can be visualized much better. Individual hairpin-like and cane-like vortices of different sizes can be discerned clearly. Several of them can be seen to wrap around the LMRs.

\subsubsection{Vortex organization}

The difficulty of visualizing flows at high Reynolds numbers is a common problem because of the large range of scales involved (Tomkins \& Adrian 2003). At higher Reynolds number it is possible to visualize vortices of a particular scale by imposing a low-pass filter. A similar technique to that used by Tomkins \& Adrian (2003) is employed here. Filtering is done in the spanwise direction by performing a moving average over a distance equal to the cube height $h$. This averaging distance is chosen because the spacing of the LMRs is of order $2 h$. Figure 19 shows a vector plot of $(u, v)$ in part of the horizontal $(x, y)$ plane at $z / h=1.5$ for the original and filtered velocity fields. A constant convection velocity, taken as the spatially averaged velocity in that plane, is subtracted from the streamwise velocity component (Galilean decomposition). The filtering produces a very clear picture of an elongated LMR bordered by several pairs of counter-rotating vortex pairs, which can be interpreted as the legs of hairpin vortices cut by the plane shown. The spanwise smoothing produces a slight 'smearing' effect on the wind vectors, but that effect is clearly distinguishable from the actual vortical structures. The similarity with the results of Tomkins \& Adrian (2003) is remarkable. The streamwise alignment of several pairs of counter-rotating vortices lends support to the presence of hairpin packets.

\subsection{Conditionally averaged structures}

In the last section, flow visualization has been used to demonstrate the existence of distinct Q2 and Q4 regions, LMRs and hairpin-type vortices. Some evidence has also been presented for the organization of the vortices into coherent packets. Following Tomkins \& Adrian (2003), statistical methods are now employed to show that the vortices and LMRs are significant and to deduce their average length scales. 


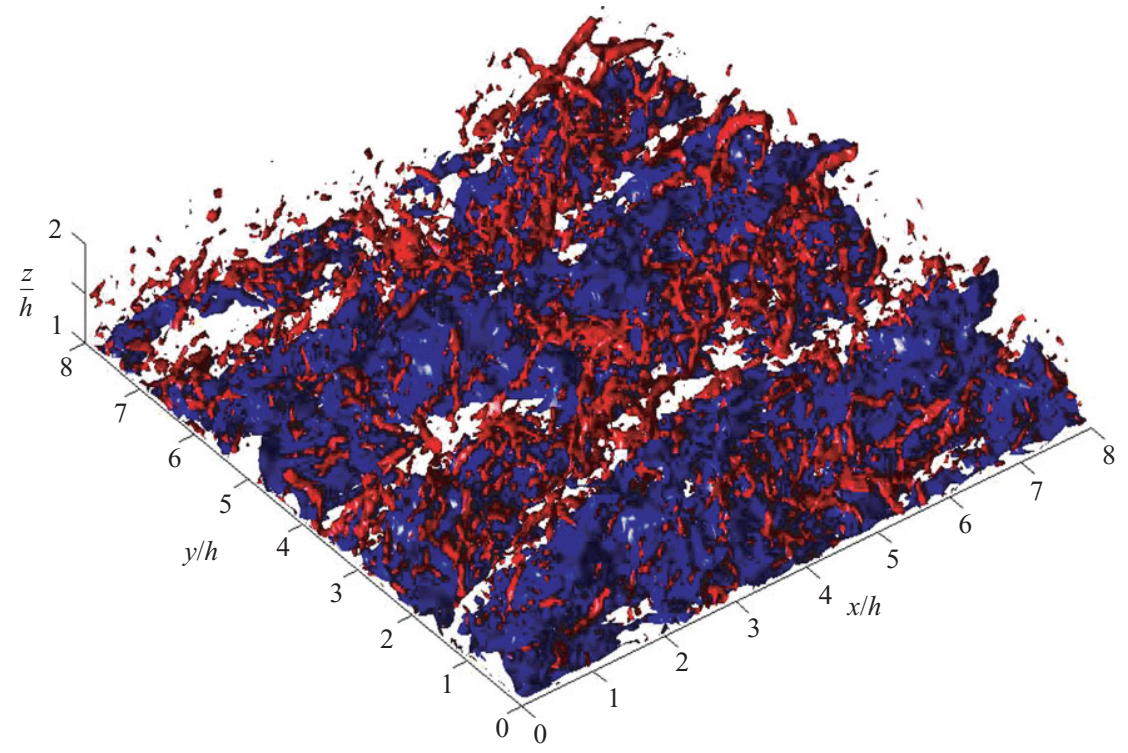

FIGURE 17. Low-speed regions (blue) and vortical structures (red) visualized by isosurface plots of negative $\lambda_{2}$ (Jeong \& Hussain 1995). Note: this figure is produced using data at half the original data resolution. A full resolution image of part of this plot may be viewed at the web address given at the end of the paper.

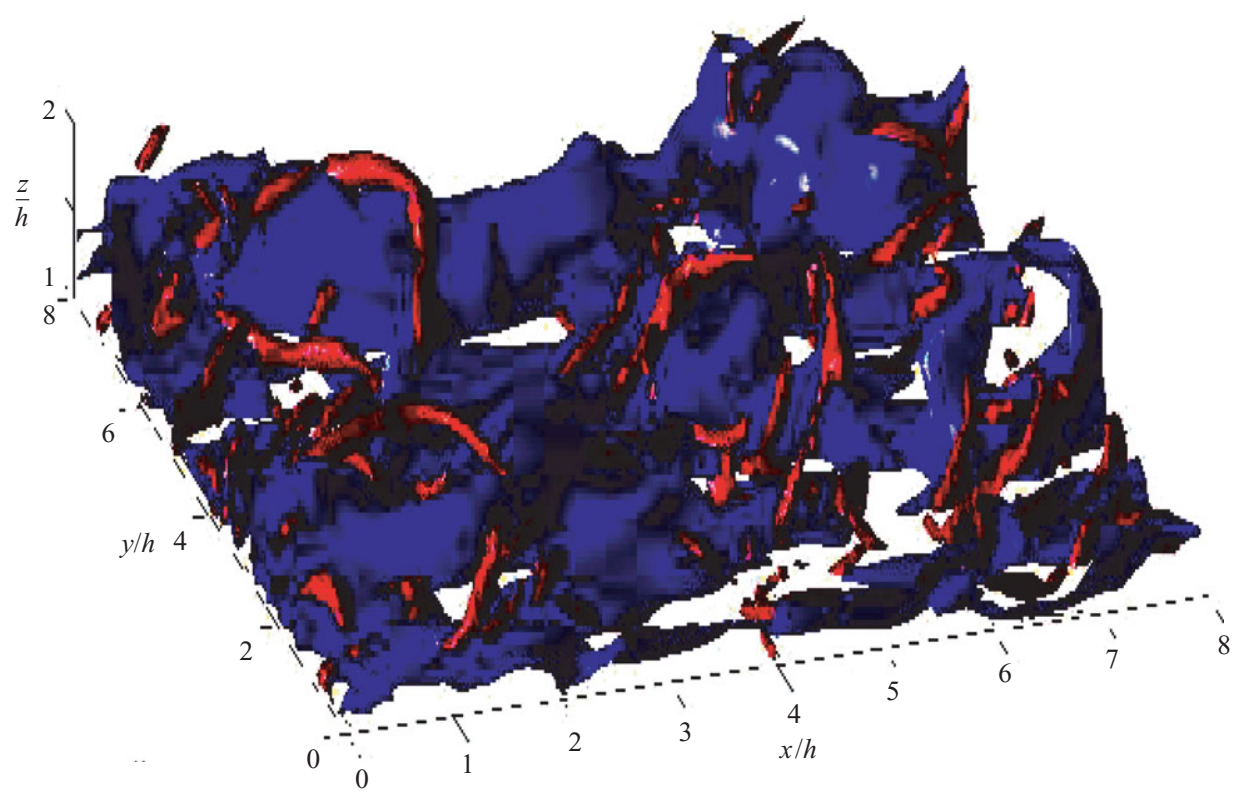

FiguRE 18. Low-speed regions (blue) and vortical structures (red) visualized by isosurface plots of negative $\lambda_{2}$, for a simulation at the lower Reynolds number $R e_{\tau}=125$.

\subsubsection{LMRs educed by linear stochastic estimation}

How predominant are the LMRs visualized in the instantaneous snapshots? Visual inspection of a large number of snapshots at widely different time intervals shows that they are extremely common, but it is important to demonstrate their importance 
(a)

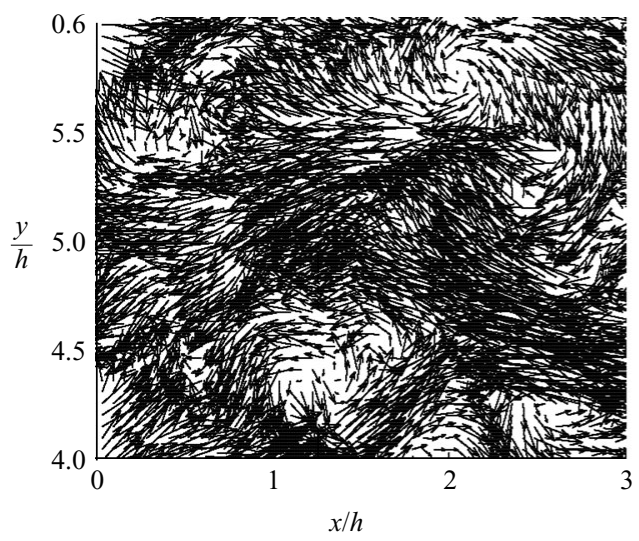

(b)

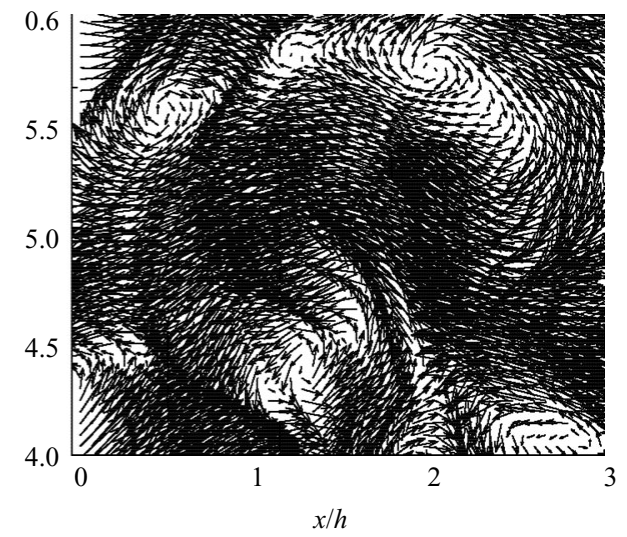

FIGURE 19. Low-pass filtering of instantaneous velocity field illustrated in part of the $(x, y)$-plane at $z=1.5 h$. (a) Original velocity field, $(b)$ filtered velocity field. Wind vectors are plotted at reduced resolution for clarity. The resulting filtered wind vector plot of $(u, v)$ reveals streamwise organization of counter-rotating vortex pairs, similar to results of Tomkins \& Adrian (2003) over smooth walls.

statistically. Tomkins \& Adrian (2003) showed that LMRs are so frequent over smooth walls that they dominate the conditional average computed given only an event of negative fluctuating streamwise velocity. They computed the conditional average using the method of linear stochastic estimation (LSE) (Adrian 1975). LSE provides a means of accurately estimating the conditional average using only unconditional data. This is generally easier than to compute the conditional average directly, and is particularly advantageous when a sufficiently large number of realizations of a trigger event is not available. The same method is now applied to the data from the present simulations.

The conditional average of the velocity field $u_{i}\left(x^{\prime}\right)$ at a location $x^{\prime}$ in space given some event $E_{j}(x)$ specified at location $x$ may be written as $\left\langle u_{i}\left(x^{\prime}\right) \mid E_{j}(x)\right\rangle$, where $j$ runs from 1 to $M$, the number of event data. The linear stochastic estimate $\hat{u}_{i}\left(x^{\prime}\right)$ of this conditional average is then given by (Adrian, 1975)

$$
\hat{u}_{i}\left(x^{\prime}\right)=A_{i j}\left(x^{\prime}\right) E_{j}(x),
$$

where the coefficients $A_{i j}$ are chosen so as to minimize the mean-square error in the estimate. This minimization gives

$$
\left\langle E_{j}(x) E_{k}(x)\right\rangle A_{i j}=\left\langle u_{i}\left(x^{\prime}\right) E_{k}(x)\right\rangle .
$$

Note from (4.1) and (4.2) that the averaging is now contained in the coefficients $A_{i j}$ and that it is an unconditional average.

Here the event vector $E_{j}(x)$ is chosen to be simply a negative fluctuating streamwise velocity, $u_{1}<0$. This then gives

$$
\hat{u}_{i}\left(x^{\prime}\right)=A_{i 1} u_{1}
$$

with

$$
\left\langle u_{1}^{2}(x)\right\rangle A_{i 1}=\left\langle u_{i}\left(x^{\prime}\right) u_{1}(x)\right\rangle
$$

and so the LSE coefficients reduce to the two-point correlation. 

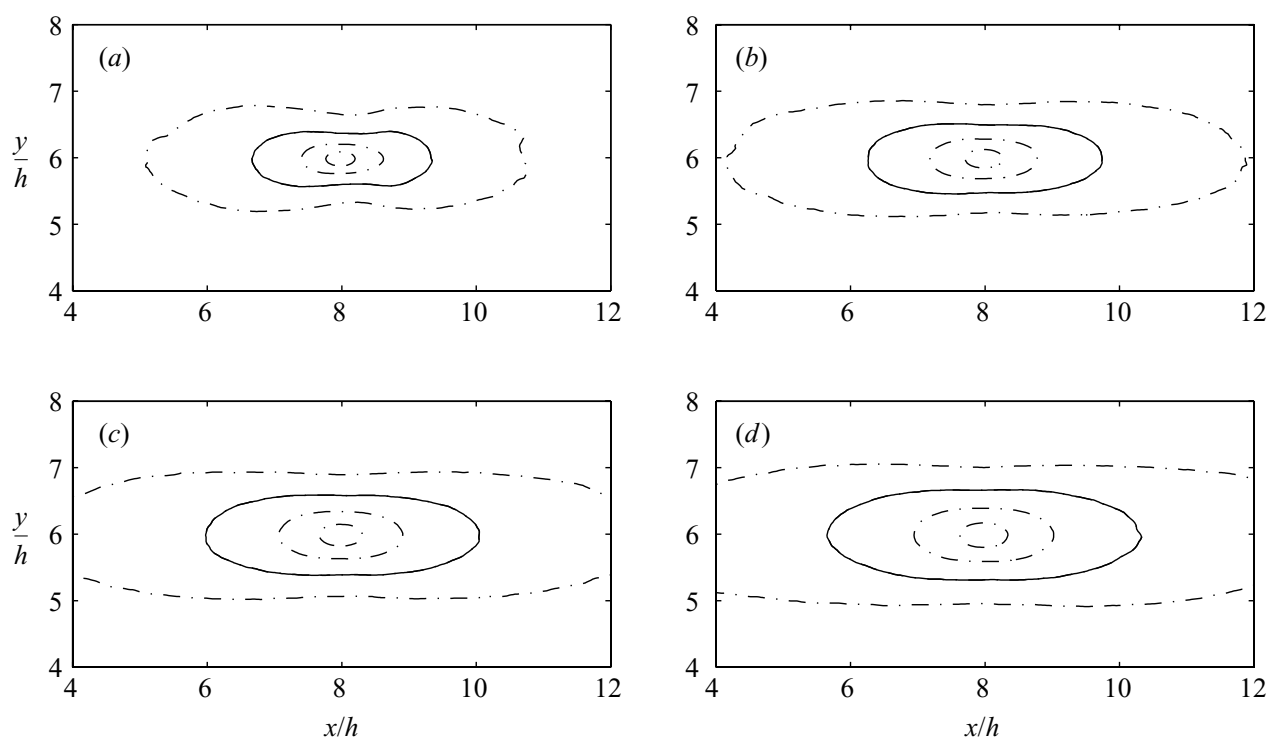

FIGURE 20. Countour plots of the conditional streamwise velocity field given a point event of negative fluctuating streamwise velocity, estimated using linear stochastic estimation (LSE). The velocities are normalized by their maximum numerical value. $(a) z=1.25 h,(b) z=1.5 h$, (c) $z=1.75 h,(d) z=2.0 h$. Plotted contours have values $-0.2,-0.4,-0.6$ and -0.8 , with the -0.4 contour shown as a solid line. These plots show elongated regions of momentum deficit whose streamwise and lateral extents increase with height.

The LSE estimate of the conditionally averaged velocity field $\hat{u}\left(x^{\prime}\right)$ given this event are computed at different heights $z$. The contour plots in figure 20 show that the conditional average given an event of negative fluctuating streamwise velocity at a point is an elongated region of low streamwise momentum.

The length and width of the 0.4 contour, scaled and plotted in wall units, are shown in figure $21(a)$. The origin of $z^{+}$for the present roughness is taken at $z=d$, where $d=0.84 h$ (conclusions do not change qualitatively if $d=0.6 h$ is used). The plots also show corresponding smooth-wall data from Tomkins \& Adrian (2003). The width scales linearly with height almost from the top of the roughness to $z^{+}=950$. The length scales approximately linearly from $z^{+}=200$ to $z^{+}=950$ (corresponding to $z=1.25 \mathrm{~h}$ to about $z=2.75 \mathrm{~h}$ ). The width of the LMRs from the smooth-wall data practically collapse with the present rough-wall data. However, the corresponding lengths of the LMRs over the smooth wall are generally larger than over the present roughness, with the difference being largest close to the surfaces and decreasing rapidly with distance from the surfaces.

The ratio of length to width of the $u=0.4$ contour is shown as a function of $z^{+}$ in figure 21(b). This ratio lies between the values of 2.1 and 3.7. The ratio is less than over smooth walls, where it is between 4 and 6 (Tomkins \& Adrian 2003). Therefore, in relation to their length, the LMRs are wider compared to smooth walls. The difference is largest near the surfaces (with a ratio of about 2.7 near $z^{+}=100$ ) and decrease with increasing distance from the surfaces, being relatively small (with a ratio of about 1.2 ) by about $z^{+}=450$. These results are consistent with two-point correlation measurements performed by Krogstad \& Antonia (1994) over a smooth wall and over a mesh roughness. They found that the spanwise length scales were similar over both surfaces, but that the streamwise length scales over the smooth wall 

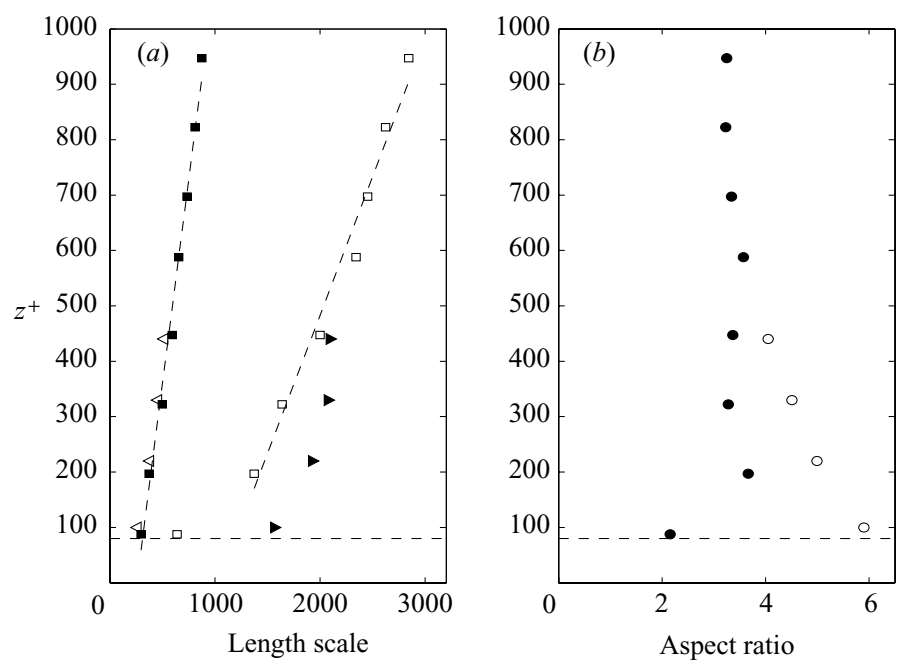

FIGURE 21. (a) Length (open squares) and width (filled squares) of the -0.4 contour of conditional streamwise velocity shown in figure 20, as a function of height. Triangles: smooth-wall data from Tomkins \& Adrian (2003). (b) Ratio of length to width of the -0.4 contours as a function of height. Filled circles: present data; open circles: smooth-wall data from Tomkins \& Adrian (2003). All length scales are scaled and plotted in wall units. The origin of $z^{+}$for the present roughness is taken at $z=d$, where $d=0.84 h$. Dashed lines indicate top of roughness.

were larger than those over the rough wall by a factor of two or more. These authors pointed out that this is consistent with the structures being inclined at a steeper angle near the wall over the rough wall compared to the smooth wall, as noted in $\S 3.5$. Similar results were also reported recently by Leonardi et al. (2004) in a DNS study on near-wall structures over square bars. They found that near-wall structures were less elongated and were larger in the spanwise direction compared to smooth walls. They attributed these changes to ejections of fluid from the cavities within the roughness, which tends to reduce streamwise coherence. This idea is supported by the early investigations of Grass (1971), who studied smooth and rough wall flows in a water channel and pointed out the strong effects of inrushes and ejections of fluid from cavities on the streaky structures over the rough surfaces. More recent flow visualizations by Grass, Stuart \& Mansour-Thehrani (1993) over roughness consisting of spheres showed that the streamwise coherence is reduced relative to a smooth wall. Ejections of low-speed fluid from within the roughness and their disruptive effect on the LMRs in the present flow has also been observed to occur intermittently in a long time sequence of snapshots.

Figure $21(b)$ also shows that the ratio $l / w$ is approximately constant from $z^{+}=200$ to $z^{+}=950(z=1.25 h$ to $z=2.75 h)$. This means that the streamwise and spanwise length scales increase roughly in the same proportion with distance from the wall in that range, so that the overall shape of the LMRs is preserved on average.

\subsubsection{Vortical structures educed by conditional averaging}

In the last section the dominant large-scale structures of the flow (LMRs) were educed by specifying a negative streamwise velocity fluctuation. To educe the dominant smaller-scale structures, the specification of local information is needed. Tomkins \& Adrian (2003) specify a local minimum velocity below a threshold set at $75 \%$ of the 
(a)

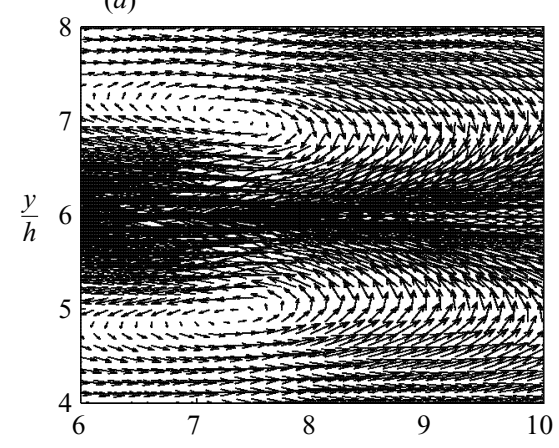

(c)

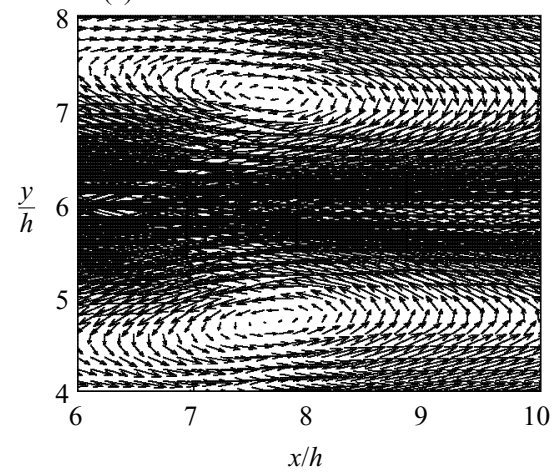

(b)

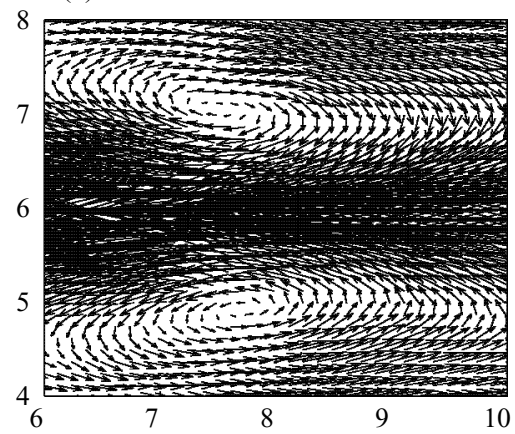

$(d)$

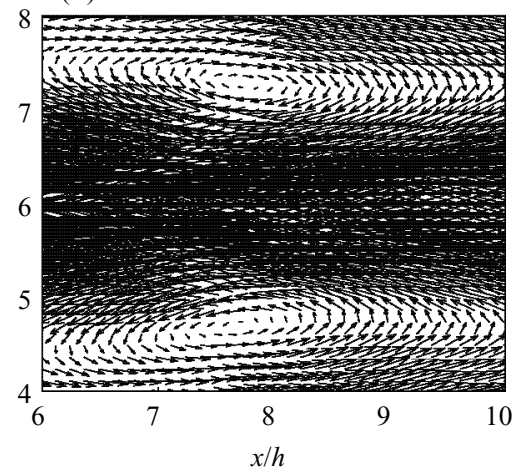

FIGURE 22. Wind vector plots of conditionally averaged fluctuating velocity field $(u, v)$ given a local minimum streamwise velocity below a negative velocity threshold. In the $(x, y)$-plane at $(a) z=1.25 h,(b) z=1.5 h,(c) z=1.75 h,(d) z=2.0 h$.

local mean velocity. The same trigger event is adopted here to perform conditional averaging of the data. This is done in the following steps: (i) at a particular height $z$ the locations of points where the event occurs are identified in each snapshot, (ii) the coordinates are horizontally shifted so as to centre on those locations successively, making use of the periodic boundary conditions in the process, (iii) a composite field is obtained by summing the velocity fluctuations field over all such locations and over all snapshots, (iv) this process is repeated at different heights. About 400 snapshots were used, equally spaced in time over a total duration of 100 eddy turnover times.

Figure 22 shows fluctuating velocity vectors $(u, v)$ of the conditional velocity field (normalized by $\left.u_{\tau}\right)$ in the $(x, y)$-plane at $z=1.25 h, 1.5 h, 1.75 h$ and $2.0 h$. At each height there is a pair of counter-rotating vortices with an elongated LMR between. As height increases, the spacing $w$ between the cores of the vortex legs increases. This is plotted in wall units in figure 23, with the origin of $z^{+}$being at $z=d$, where $d$ is again taken to be $0.84 h$ (but with qualitatively similar results with $d=0.6 h$ ). Figure 23 shows once again that the characteristic length scale of the structure grows linearly with distance from the roughness top. This confirms and lends quantitative support to the observations of Grass et al. (1991). The width $w^{+}$of the conditional eddy increases from 1050 at $z^{+}=200$ to 1900 at $z^{+}=1080(w=2.1 h$ at $z=1.25 h$ to $w=3.8 h$ at $z=3 h$ ). Comparing with the eddy sizes from the smooth-wall data of 


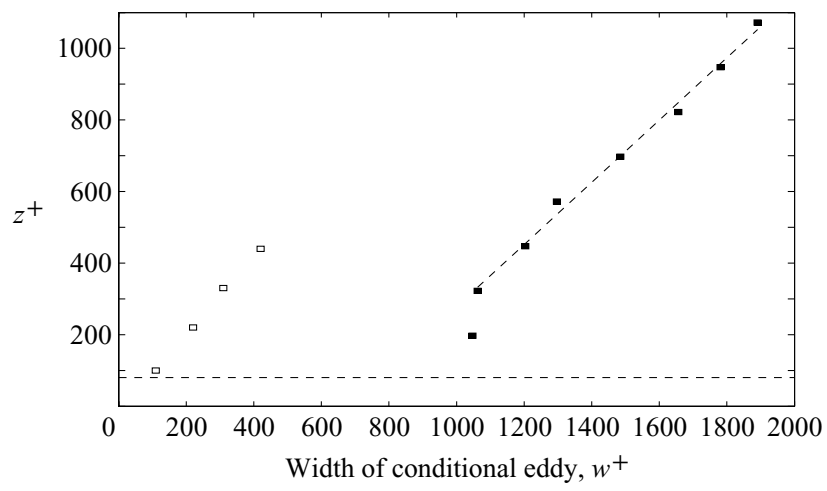

Figure 23. Width of the conditional eddy depicted in figure 22 as a function of height. Filled squares: present data; open squares: smooth-wall data from Tomkins \& Adrian (2003). All length scales are scaled and plotted in wall units. The origin of $z^{+}$for the present roughness is taken at $z=d$, where $d=0.84 h$. Dashed lines indicate top of roughness.

Tomkins \& Adrian 2003 (plotted as open squares in figure 23), the present structures can be seen to be much larger, with the smooth-wall vortex sizes being in the range $110<w^{+}<420$. This large difference is related to the different mechanisms by which vortices are generated near the wall in the two cases. The fact that the width of the vortex is $2 h$ (i.e. a simple multiple of the cube size) near the roughness suggests that they are generated by vortex shedding from the cubes. This vortex shedding seeds the flow with vortices of that scale (which are much larger than those produced in the near-wall region of smooth walls), and the vortices then grow in proportion to their distance from the wall.

Figure 24 shows the locations of Q2 and Q4 events compared with the velocity vector plot of the conditionally averaged velocity field $u$ in an $(x, y)$ and $(y, z)$ plane, for an event specified at a fixed wall normal distance of $z=1.5 h$. This clearly shows ejection events in the region between the vortex legs and sweep events on the other sides of the vortex legs. Together with the LMRs between the vortex legs, this suggests a triple association between these three coherent structures: LMRs, hairpin vortices and Q2/Q4 regions. However, the conditional averaging method is unable to establish a quantitative statistical association between them. For example, it does not tell us what fraction of the Q2/Q4 events is associated with the hairpin vortices. Moreover, it is difficult to establish a correspondence between the sizes of the LMRs and those of the vortices. Part of the problem lies with the methodological difficulties in deducing both eddy sizes and LMR sizes from the conditional averages it is difficult to characterize the precise location of the vortex cores, and to ensure consistent thresholding when applying the LSE technique. If one simply divides the vortex widths (obtained by conditional averaging) in figure 23 by the LMR widths (obtained by LSE) in figure 21(a), one finds that the vortex width (as defined here) is between two and three times the width of the LMR at a corresponding distance from the wall. Taken by itself, and remembering that the LMR widths in figure $21(a)$ are defined in terms of the -0.4 contour of the LSE plots in figure 20 , this is consistent with the picture of the LMRs being produced by back-induction within the vortex legs. However, the ratio of the vortex width to the LMR width in the present rough-wall DNS is noticeably larger than the corresponding ratio obtained in the smooth-wall experiment of Tomkins \& Adrian (2003). This reflects the fact that the 
(a)

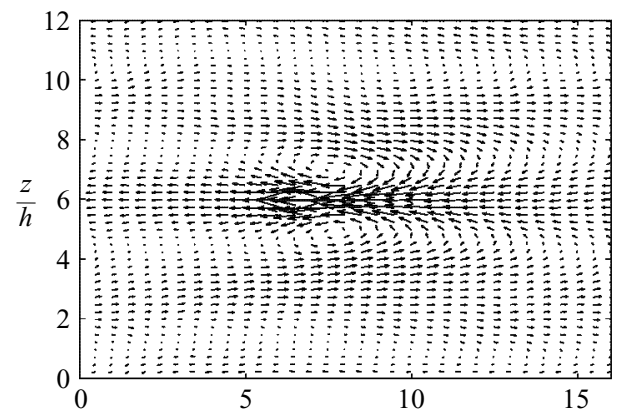

(c)

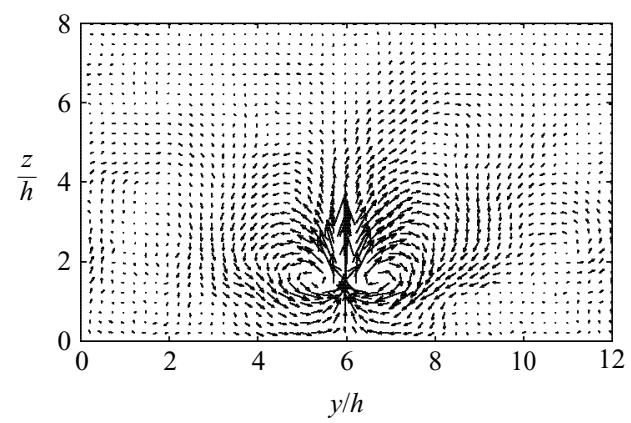

(b)

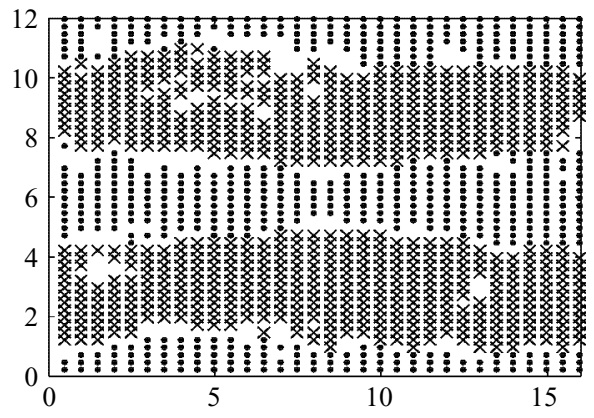

$(d)$

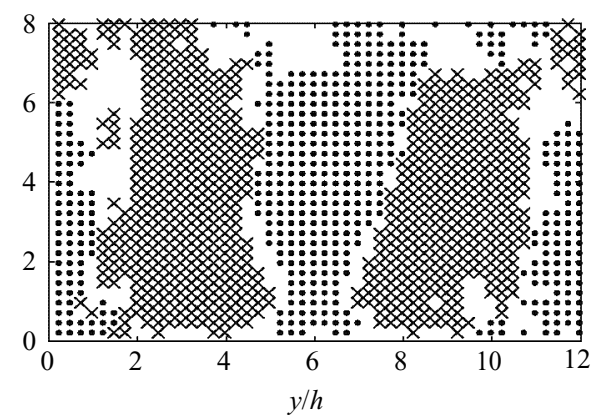

FIGURE 24. (a) Wind vector plot of conditionally averaged velocity field in the $(x, y)$-plane at $z=1.5 h$ given a local streamwise velocity minimum event specified at $z=1.5 h$. (b) Spatial distribution of Q2 and Q4 events in the same plane. Dots: Q2 events; crosses: Q4 events. (c) Wind vector plot of conditionally averaged velocity field in the $(y, z)$-plane at $x=8 h$. (d) Spatial distribution of Q2 and Q4 events in the same plane as in $(c)$. Dots: Q2 events; crosses: Q4 events.

present hairpin-like vortices are larger than their smooth-wall counterparts, whereas the sizes of the corresponding LMRs are very similar. This issue is discussed at the end of $\S 5.1 .2$.

The three-dimensional structure of the conditional eddy may be visualized by plotting negative isosurfaces of the $\lambda_{2}$ vortex identifier (Jeong \& Hussain 1995). This is plotted in figure 25 using two different thresholds of $\lambda_{2}=-0.2$ and $\lambda_{2}=-0.4$ for an event specified at $z=1.5 \mathrm{~h}$. Using the first threshold reveals more structural details, and the general structure of the conditional vortex in figure $25(a)$ is similar to the three-dimensional vortex structure educed by Zhou et al. (1999) using linear stochastic estimation, with a bridge linking the vortex pair similar to a vortex head, and the vortex core extending downstream almost horizontally beyond that bridge. Using the higher negative threshold isolates the most significant features, and reveals a structure that has the general appearance of a hairpin vortex similar to those observed in the boundary layer over smooth walls. Note that the vortex cores visualized by the $\lambda_{2}$ method do not coincide exactly with the centres of circulation on the corresponding wind vector plots (Jeong \& Hussain 1995). As a consequence the conditional eddy visualized in figure 25 appears smaller than in figure 24 . This three-dimensional visualization of the conditional vortex structure gives strong evidence that hairpinlike vortices are predominant flow structures over the present type of roughness. 


\section{Discussion and conclusions}

\subsection{Relation of present results to existing paradigms}

We conclude this paper by an examination of the implication of the results in the light of a number of current paradigms in wall-bounded flows.

\subsubsection{The attached-eddy hypothesis}

The present results share, in common with the corresponding smooth-wall results of Tomkins \& Adrian (2003), the feature that characteristic length scales associated with the coherent structures increase linearly with distance from the wall in the log region. The width of the conditional eddy scales linearly with distance from the rough surface (figure 23), as do the streamwise and spanwise dimensions of LMRs educed using LSE (figure 21a). The linear scaling of characteristic length scales is a consequence of Townsend's attached-eddy hypothesis (Townsend 1976). However, some care is needed in relating the present results to the attached-eddy hypothesis. Inspection of figure 21(a) reveals that there is an offset in the linear scaling of the LMR sizes in both the present data and that of Tomkins \& Adrian (2003), so that the effective origin of the LMRs does not correspond to the origin of the surface $(z=0$ in the smooth-wall case and $z=d$ in the rough-wall case). This is perhaps not surprising, given that the LMRs contain large-scale inactive as well as active motions (Adrian et al. 2000). Since the inactive turbulence does not scale with height (Perry et al. 1986), this may account for the constant offset on the linear scaling of the LMRs. Perhaps more surprising is the fact that the conditional eddy sizes in figure 23 also has a large offset, much larger than the corresponding offset for the smooth-wall eddies. Hence, these eddies do not have an origin at the physical origin of the surface $(z=d)$, although they are still attached in the sense that their size scales linearly from the wall (Adrian et al. 2000). In that sense, our eddies are more analogous to type-B eddies, in the terminology of Perry \& Marusic (1995). The physical reason for the offset in the scaling of the eddy sizes is related to the mechanism of their production at the rough wall. Vortex shedding by the three-dimensional roll-up of the shear layer at the leading edge of the cubes produces initial hairpin eddies with widths of the order of $2 h$. The mechanism by which these seed vortices produce larger hairpins with widths that scale with wall distance is outlined in the next subsection.

\subsubsection{The hairpin vortex packet model}

There is abundant evidence for the presence of the basic structural elements of the vortex packet model (Adrian et al. 2000; Tomkins \& Adrian 2003) in the log region within the present flow. Hairpin vortices have been directly visualized in threedimensions at $R e_{\tau}=125$ (figure 18), and inferred from filtered data (figure 19) and conditional averaging at higher $R e_{\tau}=500$ (figure 25). There is also abundant direct evidence for the existence of LMRs from flow visualization (figure 16) and conditional analysis (figure 20).

Low-pass filtering of the data has also revealed quasi-streamwise organization of a number of such vortices (figure 19). Induction due to the legs of these vortices gives rise to elongated LMRs with the observed length and width. The alignment of the vortices is consistent with the vortex packet model. A mechanism by which vortex packets are produced is described in Adrian et al. (2000). First, a primary hairpin is formed from an unspecified disturbance that creates a Q2 event near the wall. The sheared mean velocity profile causes the hairpin to be stretched and intensified, so that it grows continuously from a hairpin- to an omega-shaped vortex. If it is of sufficient strength to induce a strong Q2 event, then a new hairpin is created by interaction of 

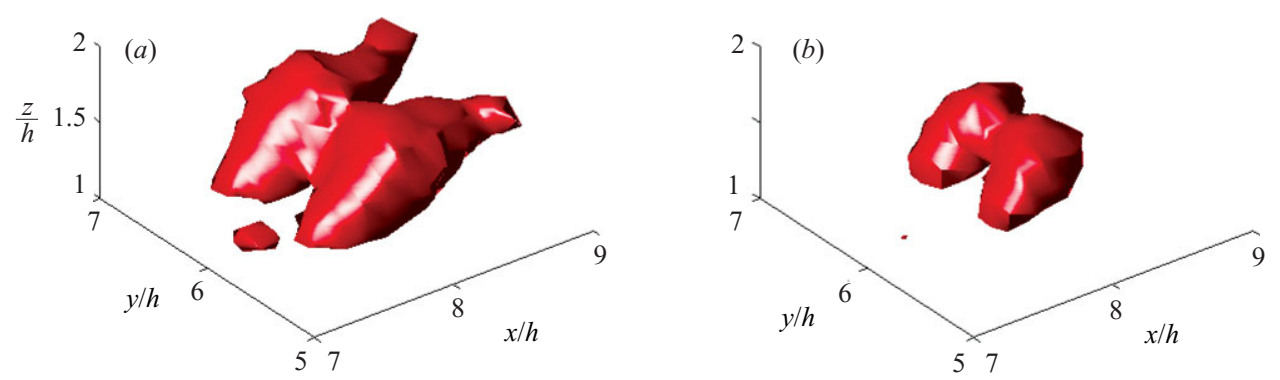

FIGURE 25. Three-dimensional structure of the conditionally averaged vortex given a local streamwise velocity minimum event specified at $z=1.5 h$. The vortex is visualized as an isosurface of negative $\lambda_{2}$ using two different thresholds: $(a) \lambda_{2}=-2.0,(b) \lambda_{2}=-4.0$.

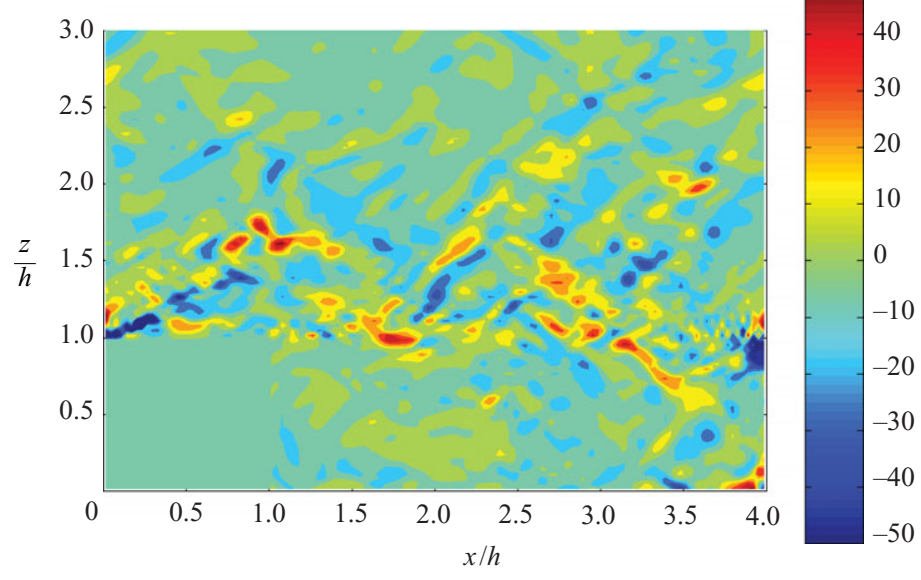

FIGURE 26. Instantaneous snapshot showing contours of spanwise vorticity $\omega_{y}$ in an $(x, z)$-plane through the middle of the cube shown on the bottom left. Values of vorticity shown on the colour bar are normalized by $u_{\tau} / h$.

this Q2 event with high-speed fluid upstream. The secondary hairpin grows in time until it also produces a tertiary hairpin, and so on. This mechanism depends only on the presence of a primary hairpin in a sheared mean flow (see also Suponitsky, Cohen \& Bar-Yoseph 2005). The primary hairpin may be created by any means that generates a Q2 event. Hence, this is an equally viable mechanism for rough walls as well as smooth walls. Indeed, evidence for vortex packets has recently been observed over natural rough surfaces such as in the atmospheric boundary layer (Hommema \& Adrian 2002) and in the bottom boundary layer of coastal oceans (Smith, Katz \& Osborn 2005).

The linear scaling of structure length scales with wall distance implies that the structures grow self-similarly. The mechanism of self-similar growth of the vortices has been studied in some detail by Tomkins \& Adrian (2003). They showed that, whilst the linear scaling of spanwise length scales may suggest self-similar growth of spanwise structure in an average sense, inspection of their data reveals that individual structures do not grow strictly self-similarly in time. Rather, they suggest the merging of vortex packets on an eddy-by-eddy basis as a mechanism by which additional scale 


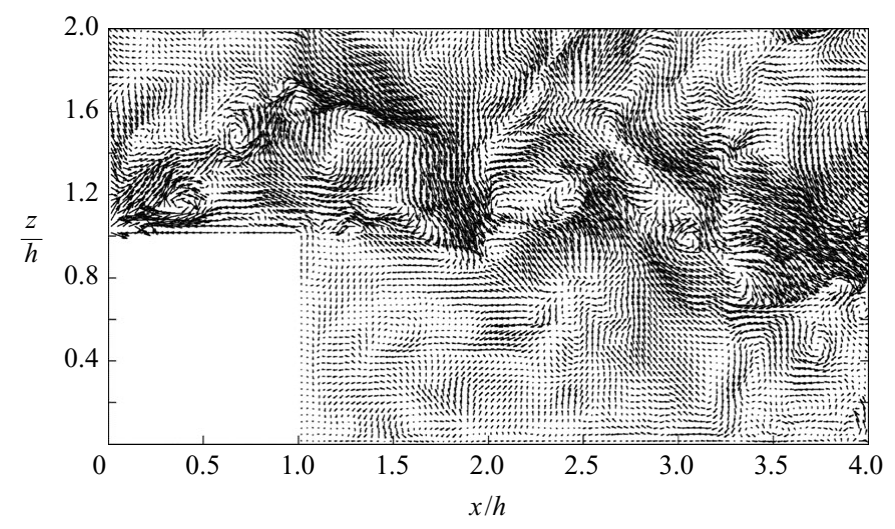

FIGURE 27. Fluctuating wind vectors $(u, w)$ corresponding to the snapshot in figure 26, and plotted in the same plane.

growth may occur. The merging takes place by a vortex reconnection mechanism as envisaged by Wark \& Nagib (1989), and may involve several different merging scenarios. Given the striking similarities of the present results with the smooth-wall case, it is not unreasonable to envisage similar growth mechanisms as proposed by Tomkins \& Adrian (2003). The crucial difference would then be that, whereas the structures in the log layer over smooth walls grow from smaller vortices generated in the viscous near-wall region, the present structures probably grow mainly from vortices shed by the obstacles. Hence, the initial scale of the vortices is set by the geometry of the obstacles. This is supported by the observation that the width of the conditional vortex is approximately $2 h$ near the top of the roughness.

Further support for this idea is provided by a recent numerical study by Hwang \& Yang (2004), who demonstrated the generation of hairpin vortices by the roll-up of the shear layer at the leading edge of a cubical obstacle in channel flow. Evidence of this vortex shedding mechanism over the cubes in the present roughness is shown by instantaneous flow visualization, examples of which are given in figures 26 and 27. Figure 26 shows contours of spanwise vorticity $\omega_{y}$ in an $(x, z)$-plane through the middle of the cube shown on the bottom left of the plot. The red and blue regions indicate high positive and negative vorticity respectively. The fluctuating wind vectors $\left(u^{\prime}, w^{\prime}\right)$ for the same snapshot are plotted in figure 27. Inspection of these plots reveals multiple vortical structures shed off the cube convecting downstream. Some of these structures spawn further vortices upstream, weaken and then decay. There is clearly a viable mechanism for generating the basic elements of the hairpin vortex packet model.

An interesting finding from the present DNS is that while the vortex sizes are much larger than over smooth walls, the LMR widths are surprisingly similar. The reason for this is an open question worthy of future investigation, but for now we briefly speculate on possible reasons. Adrian et al. (2000) pointed out that, since the back-induced flow is proportional to the vortex core circulation divided by the diameter of the hairpin, larger packets have weaker back-induction. The result is that the width of the induced LMRs decreases in relation to the size of the vortex. This is reflected in the fact that the ratio of vortex width to LMR width in the data of Tomkins \& Adrian (2003) increases by nearly a factor of two as the vortex size increases from the bottom to the top of the log region. This may be one reason why, although the hairpins in the present rough-wall data are substantially larger than the 
smooth-wall hairpins, the corresponding LMR widths at the same wall distance are very similar. Moreover, in addition to being larger than their smooth-wall counterparts, the rough-wall eddies extracted herein are in general more inclined than those over smooth walls. One consequence of this is that less lower-momentum fluid is lifted from below, which would thus tend to produce LMRs of lesser width in relation to the hairpin widths. Another possibility is that the LMRs could be dominated by more universal, passive turbulence. On this view, whilst hairpin eddies produce LMRs, not all LMRs are necessarily associated with eddies. It may be that a more complex mechanism operates that includes the hairpin vortex packet model but also other mechanisms.

\subsubsection{Outer-wall-layer similarity}

Considered solely in the light of similar findings over smooth walls, the present results would appear to offer at least qualitative support to Raupach et al.'s (1991) hypothesis of outer-layer similarity of wall-bounded flows, despite some detailed quantitative differences in the two flows. However, within the context of the broader literature it is apparent that not all types of roughness behave in the same way. The present type of roughness may be characterized as ' $\mathrm{k}$ ' type, using the terminology of Perry, Schofield \& Joubert (1969). Laboratory and DNS studies of 'd'-type roughness, consisting of two-dimensional square bars (Djenidi et al. 1999; Leonardi et al. 2004; Krogstad et al. 2005), have shown that the effect of the roughness may extend into the whole boundary layer. This hints at important differences in the dynamics of turbulent flow over three-dimensional roughness of the present kind and two-dimensional bar roughness. Djenidi et al. (1999) note the presence of low-speed streaks and vortices similar to those over smooth walls, but stress the importance of outflows from the cavities and inflows into the cavities in d-type roughness, associated with the passage of near-wall vortices. As envisaged by Perry et al. (1969), the particular twodimensional close-packed geometry of d-type roughness gives rise to stable vortices within the cavities. However, the low pressure in the cores of passing vortices triggers sequences of outflows from the cavities. Djenidi et al. (1999) point out that these sequences may persist over 10 cavities or a distance of $20 \delta$, and may explain increases in the Reynolds shear stress in the outer layer relative to smooth walls.

The present work, in common with the wind-tunnel results of Cheng \& Castro (2002), has also raised the possibility that the value of von Kármán's constant $\kappa$ may be substantially different from its smooth-wall value of around 0.41 over the present 'k-type' roughness, if one assumes that the displacement height $d$ is given by the centroid of the drag as argued by Jackson (1981). If that is true then once again the outer-wall-layer similarity hypothesis would be undermined. Another possibility is that Jackson's method for computing $d$ may not be valid for the present type of roughness. We regard this as an important open question that deserves a separate study.

\subsubsection{The canopy mixing layer analogy}

In the vicinity of the roughness there are some similarities with vegetation canopies that are worthy of further investigation. For $z / h<1.25$, there are large contributions from Q4 events to the Reynolds stress (figure 14), just as there are in the roughness sublayer over vegetation, and in mixing layers. One can envisage a possible mechanism to explain this similarity. The existence of an inflection point in the mean velocity profiles at $z=h$ (figure 5) offers the possibility that an inviscid instability that generates mixing-layer-type vortices might operate in the same way as over vegetation canopies. 
However, unlike over vegetation, the inflected profiles are associated with individual cubes, rather than with the roughness canopy as a whole, and hence their associated scales are correspondingly smaller. Hence, any generated mixing layers would be localized and not on the scale of the roughness canopy, and the corresponding eddies would be much smaller than in the roughness sublayer over vegetation canopies. This is consistent with structure above about $z=1.25 \mathrm{~h}$ looking very similar to smooth walls.

By considering the decay of two-point correlations, Castro et al. (2006) found evidence of two-scale behaviour near the top of the cubes. For example, at $z / h=1.25$ they found two integral length scales with $L_{x}=0.5 h$ for small probe separations, and $L_{x}=2.5 \mathrm{~h}$ for large separations. The larger scales pertain to the structures identified here. It is likely that the smaller length scales are associated with mixing-layer eddies produced over the cubes as envisaged above. Such structures would not be detected by the conditional sampling technique used in $\S 4.2$ because it is triggered upon local velocity minima, whereas the dominant mixing-layer vortices rotate in a sense so as to produce a strong Q4 event in between the vortex legs and thereby inducie local velocity maxima (Finnigan \& Shaw 2000).

\subsection{Concluding remarks}

A single unifying framework to understand flow over rough walls is yet to be developed, and in view of differences reported in the literature for different types of roughness, seems somewhat unlikely. In this paper we have investigated one particular type of roughness, but it has generic features - it consists of large, threedimensional bluff bodies - that make it particularly relevant for many real applications in engineering and meteorology. Fundamental information about coherent structures as revealed by the present work can be used for developing kinematic (Perry et al. 1986) and low-dimensional dynamical models (Aubry et al. 1988). Marusic (2001) incorporated the vortex packet structure of Adrian et al. (2000) into a kinematic model of the smooth-wall boundary layer developed previously by Perry \& Marusic (1995), and used it to compute structure angles and two-point correlations. He obtained results that matched experimental measurements very well, and concluded that coherent packets of vortices are indeed statistically significant structures for Reynolds stresses and transport processes. Similar kinematic and dynamic models, developed for flows over rough surfaces of practical interest would provide valuable diagnostic and predictive tools with the double advantage of being based on detailed turbulent dynamics, and being computationally very efficient compared to more conventional numerical methods such as large eddy simulations.

Animations referred to in the paper and high-resolution versions of some images can be viewed at the web page: http://www.met.rdg.ac.uk/bl_met/datasite/cubes/

The authors thank Professor Ian Castro and Dr Hong Cheng for kindly supplying their wind-tunnel data. We also thank Dr Lois Steenman-Clark, Dr Neil Stringfellow and the staff at the UK National HPC Service (CSAR) for valuable support with supercomputing. O. C. gratefully acknowledges funding from UWERN and NERC, grant number DST/26/39.

\section{REFERENCES}

Abe, H., Kawamura, H. \& Matsuo, Y. 2001 DNS of a fully developed turbulent channel flow with respect to the Reynolds number dependence. Trans. ASME: J. Fluids Engng 123, 382-393. 
AdRIAN, R. J. 1975 On the role of conditional averages in turbulence theory. In Turbulence in Liquids (ed. J. L. Patterson \& G. K. Zakin), pp. 323-332. University of Missouri, Rolla, Missouri. Science Press.

Adrian, R. J., Meinhart, C. D. \& Tomkins, C. D. 2000 Vortex organisation in the outer region of the turbulent boundary layer. J. Fluid Mech. 422, 1-54.

Aubry, N., Holmes, P., Lumley, J. L. \& Stone, E. 1998 The dynamics of coherent structures in the wall region of a turbulent boundary layer. J. Fluid Mech. 192, 115-173.

Brown, G. L. \& Roshko, A. 1974 On density effects and large structure in turbulent mixing layers. J. Fluid Mech. 64, 775-816.

Cantwell, B. J. 1981 Organized motion in turbulent flow. Annu. Rev. Fluid Mech. 13, 437-515.

Castro, I. P., Cheng, H. \& Reynolds, R. 2006 Turbulence over urban-type roughness: deductions from wind tunnel measurements. Boundary-Layer Met. 118, 109-131.

Cheng, H. \& Castro, I. P. 2002 Near wall flow over urban-like roughness. Boundary-Layer Met. 104, 229-259.

Coceal, O., Thomas, T. G. Castro, I. P. \& Belcher, S. E 2006 Mean flow and turbulence statistics over groups of urban-like cubical obstacles. Boundary-Layer Met. 121, 491-519.

Djenidi, L., Elavarasan, R. \& Antonia, R. A. 1999 The turbulent boundary layer over transverse square cavities. J. Fluid Mech. 395, 271-294.

Finnigan, J. J. 2000 Turbulence in plant canopies. Annu. Rev. Fluid Mech. 32, 519-572.

Finnigan, J. J. \& Shaw, R. H. 2000 A wind-tunnel study of airflow in waving wheat: an EOF analysis of the structure of the large-eddy motion. Boundary-Layer Met. 96, 211-255.

Gad-el-HaK, M. 2000 Flow Control. Passive, Active and Reactive Flow Management. Cambridge University Press.

Grass, A. J. 1971 Structural features of turbulent flow over smooth and rough boundaries. J. Fluid Mech. 50, 233-255.

Grass, A. J., Stuart, R. J. \& Mansour-Thehrani, M. 1991 Vortical structures and coherent motion in turbulent flow over smooth and rough boundaries. Phil. Trans. R. Soc. Lond. A 336, 35-65.

Grass, A. J., Stuart, R. J. \& Mansour-Thehrani, M. 1993 Common vortical structure of turbulent flows over smooth and rough boundaries. AIAA J. 31, 837-846.

Hamilton, J. M., Kim, J. \& WalefFe, F. 1995 Regeneration mechanisms of near-wall turbulence structures. J. Fluid Mech. 287, 317-348.

Hommema, S. E. \& Adrian, R. J. 2002 Packet structure of surface eddies in the atmospheric boundary layer. Boundary-Layer Met. 106, 147-170.

JACKSON, P. S. 1981 On the displacement height in the logarithmic profile. J. Fluid Mech. 111, 15-25.

Jeong, J. \& Hussain, F. 1995 On the identification of a vortex. J. Fluid Mech. 285, 69-94.

JimeneZ, J. 2004 Turbulent flows over rough walls. Annu. Rev. Fluid Mech. 36, 173-196.

Jimenez, J. \& Pinelli, A. 1999 The autonomous cycle of near-wall turbulence. J. Fluid Mech. 389, 335-359.

Hwang, J.-Y. \& YANG, K.-S. 2004 Numerical study of vortical structures around a wall-mounted cubic obstacle in channel flow. Phys. Fluids 16, 2382-2394.

Kim, H. T., KLINE, S. J. \& ReYnolds, W. C. 1971 The production of turbulence near a smooth wall in a turbulent boundary layer. J. Fluid Mech. 50, 133-160.

Kim, H. T., Moin, P. \& Moser, R. 1987 Turbulence statistics in fully devloped channel flow at low Reynolds number. J. Fluid Mech. 177, 133-166.

Klebanoff, P. S. 1954 Characteristics of turbulence in a boundary layer with zero pressure gradient. NACA TN 3178.

Kline, S. J., Reynolds, W. C., Schraub, F. A. \& Runstaadler, P. W. 1967 The structure of turbulent boundary layers. J. Fluid Mech. 30, 741-773.

Krogstad, P. A. Andersson, H. I., Bakken, O. M. \& Ashrafian, A. 2005 An experimental and numerical study of channel flow with rough walls. J. Fluid Mech. 530, 327-352.

Krogstad, P. A. \& Antonia, R. A. 1994 Structure of turbulent boundary layers on smooth and rough walls. J. Fluid Mech. 277, 1-21.

Krogstad, P. A. \& Antonia, R. A. 1999 Surface roughness effects in turbulent boundary layers. Exps. Fluids 27, 450-460.

Krogstad, P. A., Antonia, R. A. \& Browne, L. W. B. 1992 Comparison between rough- and smooth-wall turbulent boundary layers. J. Fluid Mech. 245, 599-617. 
Leonardi, S., Orlandi, P., Djenidi, L. \& Antonia, R. A. 2004 Structure of turbulent channel flow with square bars on one wall. Intl J. Heat Fluid Flow 25, 384-392.

Leonardi, S., Orlandi, P., Smalley, R. J., Djenidi, L. \& Antonia, R. A. 2003 Direct numerical simulations of turbulent channel flow with transverse square bars on one wall. J. Fluid Mech. 491, 229-238.

MaruSIC, I. 2001 On the role of large-scale structures in wall turbulence. Phys. of Fluids 13, 735-743.

Moin, P. \& Mahesh, K. 1998 Direct numerical simulation: a tool in turbulence research. Annu. Rev. Fluid Mech. 30, 539-578.

Panton, R. L. 2001 Overview of the self-sustaining mechanisms of wall turbulence. Prog. in Aerospace Sci. 37, 341-383.

PaO, T. H. 1965 Structure of turbulent velocity and scalar fields at large wave numbers. Phys. Fluids 8, 1063.

Perry, A. E., Henbest, S. M. \& Chong, M. S. 1986 A theoretical and experimental study of wall turbulence. J. Fluid Mech. 165, 163-199.

Perry, A. E. \& Marusic, I. 1995 A wall-wake model for the turbulence structure of boundary layers. Part 1. Extension of the attached eddy hypothesis. J. Fluid Mech. 298, 361-388.

Perry, A. E., Schofield, W. H. \& Joubert, P. N. 1969 Rough-wall turbulent boundary layers. J. Fluid Mech. 37, 383-413.

Pokrajac, D., Finnigan, J. J., Manes, C., McEwan, I., Nikora, V. 2006 On the definition of the shear velocity in rough bed open channel flows. Proc. Intl Conference on Fluvial Hydraulics River Flow 2006 Lisbon (ed. R. Ferreira, E. Alves, J. Leal \& A. Cardoso). Taylor \& Francis.

Pope, S. B. 2000 Turbulent Flows. Cambridge University Press.

RaupaCH, M. R. 1981 Conditional statistics of Reynolds stress in rough-wall and smooth-wall turbulent boundary layers. J. Fluid Mech. 108, 363-382.

Raupach, M. R., Antonia, R. A. \& Rajagopalan, S. 1991 Rough-wall turbulent boundary layers. Appl. Mech. Rev. 44, 1-25.

Raupach, M. R., Finnigan, J. J. \& Brunet, Y. 1996 Coherent eddies and turbulence in vegetation canopies: the mixing layer analogy. Boundary-Layer Met. 78, 351-382.

Robinson, S. K. 1991 Coherent motions in the turbulent boundary layer. Annu. Rev. Fluid Mech. 23, 601-639.

Rogers, M. M. \& Moser, R. D. 1992 The three-dimensional evolution of a plane mixing layer: the Kelvin-Helmholz rollup. J. Fluid Mech. 243, 183-226.

Rotach, M. W. 1993 Turbulence close to a rough urban surface, Part I: Reynolds stress. BoundaryLayer Met. 65, 1-28.

Smith, W. A. M. N., Katz, J. \& Osborn, T. R. 2005 On the structure of turbulence in the bottom boundary layer of the coastal ocean. J. Phys. Oceangr. 35 (1), 72-93.

Spalart, P. R. 1988 Direct simulation of a turbulent boundary layer up to $R_{\theta}=1410$. J. Fluid Mech. 187, 61-98.

Suponitsky, V., COHEN, J. \& BAR-YosePH, P. Z. 2005 The generation of streaks and hairpin vortices from a localized vortex disturbance embedded in unbounded uniform shear flow. J. Fluid Mech. 535, 65-100.

Tennekes, H. \& Lumley, J. L. 1972 A First Course in Turbulence. The MIT Press.

Tном, A. S. 1971 Momentum absorption by vegetation. Q. J. R. Met. Soc. 97, 414-428.

Tomkins, C. D. \& AdRian, R. J. 2003 Spanwise structure and scale growth in turbulent boundary layers. J. Fluid Mech. 490, 37-74.

Townsend, A. A. 1976 The Structure of Turbulent Shear Flow. Cambridge University Press.

Wallace, J. M., Eckelman, H. \& Brodkey, R. S. 1972 The wall region in turbulent shear flow. J. Fluid Mech. 54, 39-48.

WARK, C. E. \& NAGIB, H. M. 1989 Relation between outer structures and wall-layer events in boundary layers with and without manipulation. In Proc. 2nd IUTAM Symp. on Structure of Turbulence and Drag Reduction, Zurich, Switzerland, pp. 467-474, (ed. A. Gyr). Springer.

Yao, Y. F., Thomas, T. G., Sandham, N. D. \& Williams, J. J. R. 2001 Direct numerical simulation of turbulent flow over a rectangular trailing edge. Theoret. Comput. Fluid Dyn. 14, 337-358.

Zhou, J., Adrian, R. J., Balachandar, S. \& Kendall, T. M. 1999 Mechanisms for generating coherent packets of hairpin vortices in channel flow. J. Fluid Mech. 387, 353-396. 